\title{
Genetic Variation Among Vegetative Compatibility Groups of Fusarium oxysporum f. sp. cubense Analyzed by DNA Fingerprinting
}

\author{
S. Bentley, K. G. Pegg, N. Y. Moore, R. D. Davis, and I. W. Buddenhagen
}

First author: Cooperative Research Centre for Tropical Plant Pathology, The University of Queensland, St. Lucia, Queensland 4072, Australia; second and fourth authors: Queensland Horticulture Institute, Queensland Department of Primary Industries, Indooroopilly Sciences Centre, 80 Meiers Road, Indooroopilly, Queensland 4068, Australia; third author: Farming Systems Institute, Queensland Department of Primary Industries, Indooroopilly Sciences Centre, 80 Meiers Road, Indooroopilly, Queensland 4068, Australia; and fifth author: 1012 Plum Lane, Davis, CA 95616.

Accepted for publication 18 August 1998.

\begin{abstract}
Bentley, S., Pegg, K. G., Moore, N. Y., Davis, R. D., and Buddenhagen, I. W. 1998. Genetic variation among vegetative compatibility groups of Fusarium oxysporum f. sp. cubense analyzed by DNA fingerprinting. Phytopathology 88:1283-1293.

Genetic variation within a worldwide collection of 208 isolates of $F u$ sarium oxysporum $\mathrm{f}$. sp. cubense, representing physiological races 1, 2, 3, and 4 and the 20 reported vegetative compatibility groups (VCGs), was analyzed using modified DNA amplification fingerprinting. Also characterized were 133 isolates that did not belong to any of the reported VCGs of $F$. oxysporum f. sp. cubense including race 3 isolates from a Heliconia species and isolates from a symptomatic wild banana species growing in the jungle in peninsular Malaysia. The DNA fingerprint patterns were generally VCG specific, irrespective of geographic or host origin. A total

to the 20 reported VCGs, and 14 new genotypes were identified among the isolates that did not belong to any of the existing VCGs. DNA fingerprinting analysis also allowed differentiation of nine clonal lineages within F. oxysporum f. sp. cubense. Five of these lineages each contained numerous closely related VCGs and genotypes, and the remaining four lineages each contained a single genotype. The genetic diversity and geographic distribution of several of these lineages of $F$. oxysporum $\mathrm{f}$. sp. cubense suggests that they have coevolved with edible bananas and their wild diploid progenitors in Asia. DNA fingerprinting analysis of isolates from the wild pathosystem provides further evidence for the coevolution hypothesis. The genetic isolation and limited geographic distribution of four of the lineages of $F$. oxysporum f. sp. cubense suggests that the pathogen has also arisen independently, both within and outside of the center of origin of the host.
\end{abstract} of 33 different genotypes were identified within F. oxysporum f. sp. $\mathrm{cu}$ bense; 19 genotypes were distinguished among the isolates that belonged
Additional keywords: Fusarium wilt, Panama disease, Musa.
Fusarium wilt (Panama disease), caused by Fusarium oxysporum Schlechtend.:Fr. f. sp. cubense (E.F. Smith) W.C. Snyd. \& H.N. Hans. (25), is regarded as one of the most significant threats to banana (Musa spp.) production worldwide (19). Edible bananas originated in Asia and are now grown in virtually all areas located between $30^{\circ} \mathrm{N}$ and $30^{\circ} \mathrm{S}$ latitudes. Fusarium wilt has been reported from all banana-growing regions of the world except the South Pacific Islands, Somaliland, and countries bordering the Mediterranean Sea $(21,29)$.

F. oxysporum f. sp. cubense affects species of Musa and Heliconia, and strains have been classified into four physiological races based on pathogenicity to host cultivars in the field (race 1, 'Gros Michel'; race 2, 'Bluggoe'; race 3, Heliconia spp.; and race 4, Cavendish cultivars and all cultivars susceptible to races 1 and 2) (19). Races of F. oxysporum f. sp. cubense are not defined genetically, but are groups of isolates that attack differential cultivars in the field. Earlier this century, race 1 of Fusarium wilt nearly destroyed the world banana export industry, which was based on the Gros Michel cultivar. Consequently, 'Gros Michel' was replaced by Cavendish cultivars, which were resistant to race 1. Although Cavendish cultivars remain resistant to race 1, another race of $F$. oxysporum f. sp. cubense, which is designated race 4 , is capable of attacking Cavendish cultivars. Until recently, race 4 had only been recorded to cause serious losses in the subtropical regions of

Corresponding author: S. Bentley; E-mail address: S.Bentley@tpp.uq.edu.au

Publication no. P-1998-1016-01R

(c) 1998 The American Phytopathological Society
Australia, South Africa, the Canary Islands, and Taiwan (23). It is thought that Cavendish cultivars in subtropical regions were predisposed to infection due to cold-induced stress during winter (12, 15). The disease also occurs in the Philippines, where Cavendish cultivars succumb in localized areas under poor edaphic conditions. Recently, Fusarium wilt has devastated Cavendish cultivars (Grande Naine and Valery) in the Asian deep tropics in peninsular Malaysia, Sumatra, Java, and Halmahera, where no predisposing factors have been identified. If these strains were to become established in the Americas, the world export industries could be severely affected, as there is no widely accepted replacement for the Cavendish cultivars. Fusarium wilt is also a major concern to bananas and plantains, which are essential to the nutritional and economic well-being of millions of people throughout the developing world.

F. oxysporum f. sp. cubense is considered to be a highly complex pathogen. Numerous methods have been used to characterize $F$. oxysporum $\mathrm{f}$. sp. cubense including vegetative compatibility $(5,12,14$, $20,22)$, production of volatiles $(6,13,26)$, electrophoretic karyotyping $(4,11)$, random amplified polymorphic DNA (RAPD) analysis (3), and restriction fragment length polymorphism (RFLP) analysis (10). These methods have been useful for pathotype determination $(17,18)$, for which no reliable small-plant pathogenicity test currently exists. The use of tissue culture-derived plantlets to ascertain host-pathogen responses is generally restricted to endemic isolates and has given inconsistent results. Field testing is expensive and inefficient because of the limited number of strains in any given field.

Various genetic marker systems can be used to determine genetic diversity among different isolates within a species. Since $F$. oxysporum f. sp. cubense is a haploid asexual pathogen, arbitrary 
primer techniques such as RAPD (33) and DNA amplification fingerprinting (DAF) (8) can be used effectively. Preliminary RAPD and DAF analysis of $F$. oxysporum f. sp. cubense divided isolates into two major groups, and the genetic relationships among the vegetative compatibility groups (VCGs) were determined (1, $2,3)$. The aims of this work were to (i) assess the genetic variation among isolates within each VCG and between different VCGs of the pathogen, (ii) determine the efficacy of DNA fingerprinting analysis to predict putative VCGs among uncharacterized isolates, and (iii) delineate clonal lineages within $F$. oxysporum f. sp. cubense. The racial structure and possible origins of the pathogen are also discussed. To our knowledge, this study involves the first molecular genetic analysis of isolates from a Heliconia species and from a symptomatic wild banana species growing in a native situation.

\section{MATERIALS AND METHODS}

Fungal isolates. Two hundred and eight isolates of $F$. oxysporum f. sp. cubense from a worldwide collection and many different host genotypes, representing races $1,2,3$, and 4 and the 20 reported VCGs, were examined (Table 1). An additional 133 isolates of unknown VCG were also characterized by DNA fingerprinting

TABLE 1. Isolates of Fusarium oxysporum f. sp. cubense analyzed in this study

\begin{tabular}{|c|c|c|c|c|c|c|c|c|c|}
\hline $\begin{array}{l}\text { Accession } \\
\text { number }{ }^{\mathrm{w}}\end{array}$ & $\begin{array}{c}\text { Geographic } \\
\text { origin }\end{array}$ & $\begin{array}{l}\text { Host origin } \\
\text { and genotype }\end{array}$ & $\begin{array}{l}\text { Donor or } \\
\text { collector }^{y}\end{array}$ & $\mathrm{DFG}^{\mathrm{z}}$ & $\begin{array}{l}\text { Accession } \\
\text { number }\end{array}$ & $\begin{array}{c}\text { Geographic } \\
\text { origin }\end{array}$ & $\begin{array}{l}\text { Host origin } \\
\text { and genotype }\end{array}$ & $\begin{array}{l}\text { Donor or } \\
\text { collector }^{\mathrm{y}}\end{array}$ & $\mathrm{DFG}^{\mathrm{Z}}$ \\
\hline VCG 0120 & & & & & THAI4-2 & Thailand & Kluai Namwa & $\mathrm{f}$ & $\mathrm{V}$ \\
\hline 22615 & Byron Bay, Australia & Cavendish (AAA) & $\mathrm{a}$ & I & THAI10 & Thailand & Kluai Namwa & f & $\mathrm{V}$ \\
\hline 23486 & Wamuran, Australia & Cavendish & $\mathrm{a}$ & I & THAI19-1 & Thailand & Kluai Namwa & f & $\mathrm{V}$ \\
\hline 23516 & Wamuran, Australia & Inarnibal (AA) & $\mathrm{a}$ & I & THAI20-2 & Thailand & Kluai Namwa & f & $\mathrm{V}$ \\
\hline 23539 & Beerwah, Australia & Lady finger (AAB) & a & I & THAI21 & Thailand & Kluai Namwa & g & V \\
\hline 23550 & Wamuran, Australia & Musa jackeyi & $\mathrm{a}$ & I & F9129 & Taiwan & Latundan & d & $\mathrm{V}$ \\
\hline 23551 & Wamuran, Australia & SH3362 (AA) & a & I & RP19(DAVAO) & )Philippines & Silk (AAB) & $\mathrm{c}$ & V \\
\hline 23598 & Wamuran, Australia & Cavendish & a & I & $\mathrm{RP} 20(\mathrm{Ph} 2)$ & Philippines & Latundan & $\mathrm{c}$ & $\mathrm{V}$ \\
\hline 23599 & Wamuran, Australia & Cavendish & a & I & RP21 (PhL1) & Philippines & Latundan & $\mathrm{c}$ & $\mathrm{V}$ \\
\hline 23607 & Wamuran, Australia & SH3362 & a & I & RP22 (PhL2) & Philippines & Latundan & $\mathrm{c}$ & V \\
\hline MD401 & Wamuran, Australia & Cavendish & a & I & RP24 (T1) & Taiwan & Gros Michel & $\mathrm{c}$ & $\mathrm{V}$ \\
\hline N5631 & Landsborough, Australia & Cavendish & a & I & VCG 0124/5 & & & & \\
\hline$-42 \mathrm{~F}$ & Wamuran, Australia & Cavendish & a & I & THAI13 & Thailand & Kluai Namwa & $\mathrm{f}$ & IV \\
\hline W91-307 & Eungella, Australia & Lady finger & $\mathrm{a}$ & I & RP36 (MW5) & Malawi & Zambia (ABB) & $\mathrm{c}$ & IV \\
\hline W91-345 & Mullumbimby, Australia & Lady finger & $\mathrm{a}$ & I & RP37 (MW9) & Malawi & Zambia & $\mathrm{c}$ & IV \\
\hline 23987 & Wamuran, Australia & Cavendish & $\mathrm{a}$ & I & RP38 (MW15) & Malawi & Harare (ABB) & $\mathrm{c}$ & IV \\
\hline INDO14 & West Java, Indonesia & Pisang Ambon Putih (AAA) & $\mathrm{b}$ & I & RP39(MW61) & Malawi & Harare & $\mathrm{c}$ & IV \\
\hline RP1 (STGM1) & Costa Rica & Gros Michel (AAA) & $\mathrm{c}$ & I & RP40 (MW68) & Malawi & Kholobowa (ABB) & $\mathrm{c}$ & IV \\
\hline RP2 (STGM2) & Costa Rica & Gros Michel & $\mathrm{c}$ & I & RP41 (CVA) & United States & Apple (AAB) & $\mathrm{c}$ & IV \\
\hline RP3 (FCJ7) & Jamaica & Lacatan (AA) & $\mathrm{c}$ & I & VCG 0124 & & & & \\
\hline RP4 (NH) & Natal, South Africa & Cavendish cv. Williams (AAA) & $\mathrm{c}$ & I & 23485 & Mena Creek, Australia & Lady finger & a & IV \\
\hline RP5 (GAL1) & Canary Islands & Dwarf Cavendish (AAA) & $\mathrm{c}$ & I & 23532 & Ormeau, Australia & Lady finger & a & IV \\
\hline RP6 (SA6) & South Africa & Dwarf Cavendish & $\mathrm{c}$ & I & 23534 & Ormeau, Australia & Lady finger & a & IV \\
\hline RP3S1 & Honduras & Highgate (AAA) & $\mathrm{c}$ & I & 23536 & Brookfield, Australia & Ducasse (ABB) & $\mathrm{a}$ & IV \\
\hline RPSTH1 & Honduras & Highgate & $\mathrm{c}$ & I & 23538 & Agnes Waters, Australia & Lady finger & a & IV \\
\hline RPIC2 & Canary Islands & Dwarf Cavendish & $\mathrm{c}$ & I & 23567 & Moresby, Australia & Ducasse & $\mathrm{a}$ & IV \\
\hline F9131 & South Africa & Cavendish cv. Williams & d & I & 23603 & Tallebudgera, Australia & Lady finger & $\mathrm{a}$ & IV \\
\hline VCG 0121 & & & & & 23734 & Ormeau, Australia & Lady finger & a & IV \\
\hline F9130 & Taiwan & Cavendish & $\mathrm{d}$ & III & THAI14 & Kanjanaburi, Thailand & Kluai Namwa & $\mathrm{f}$ & IV \\
\hline RP7 (T3) & Taiwan & Cavendish & $\mathrm{c}$ & III & RP25 (A35) & Brazil & Unknown & $\mathrm{c}$ & IV \\
\hline RP8 (F9130) & Taiwan & Cavendish & $\mathrm{c}$ & III & RP26(STD1) & Honduras & Highgate & $\mathrm{c}$ & IV \\
\hline RP9 (GM) & Taiwan & Cavendish & $\mathrm{c}$ & III & RP27 (STD2) & Honduras & Highgate & $\mathrm{c}$ & IV \\
\hline RP10 (ML) & Taiwan & Cavendish & $\mathrm{c}$ & III & RP29 (FCJ3) & Jamaica & Unknown & $\mathrm{c}$ & IV \\
\hline RP11 (TBR) & Taiwan & Cavendish & $\mathrm{c}$ & III & RP30 (MW30) & Malawi & Harare & $\mathrm{c}$ & IV \\
\hline RP12 (SKC) & Taiwan & Gros Michel & $\mathrm{c}$ & III & RP31 (MW67) & Malawi & Kholobowa & $\mathrm{c}$ & IV \\
\hline VCG 0122 & & & & & RP32 (MW80) & Malawi & Harare & $\mathrm{c}$ & IV \\
\hline PHIL10 & Philippines & Cavendish cv. Grande Naine (AAA) & $\mathrm{e}$ & II & RP33 (STN2) & Nicaragua & Bluggoe (ABB) & $\mathrm{c}$ & IV \\
\hline $\mathrm{RP} 13(\mathrm{Ph} 2)$ & Philippines & Cavendish & $\mathrm{c}$ & II & RP34 (STPA2) & Tanzania & Pisang awak & $\mathrm{c}$ & IV \\
\hline RP14 (P18) & Philippines & Cavendish & $\mathrm{c}$ & II & RP35 (B1) & United States & Burro (ABB) & $\mathrm{c}$ & IV \\
\hline RP15 (P79) & Philippines & Cavendish & $\mathrm{c}$ & II & VCG 0125 & & & & \\
\hline RP16 (LAP) & Philippines & Cavendish & $\mathrm{c}$ & II & 8605 & Tallebudgera, Australia & Lady finger & a & IV \\
\hline RP17 (SABA) & Philippines & Saba (BBB) & $\mathrm{c}$ & II & 8611 & Tomewin, Australia & Lady finger & a & IV \\
\hline RP18 (PW3) & Philippines & Cavendish & $\mathrm{c}$ & II & 22468 & Currumbin, Australia & Lady finger & a & IV \\
\hline VCG 0123 & & & & & 23477 & Tallebudgera, Australia & Lady finger & a & IV \\
\hline MAL5 & Malaysia & Pisang awak (ABB) & $?$ & $\mathrm{~V}$ & 23480 & Tallebudgera, Australia & Lady finger & $\mathrm{a}$ & IV \\
\hline PHIL3 & Philippines & Latundan (AAB) & $\mathrm{e}$ & $\mathrm{V}$ & 23482 & Currumbin, Australia & Lady finger & a & IV \\
\hline PHIL8 & Philippines & Latundan & e & $\mathrm{V}$ & 23487 & Currumbin, Australia & Lady finger & a & IV \\
\hline PHIL13 & Philippines & Latundan & $\mathrm{e}$ & $\mathrm{V}$ & 23488 & Tallebudgera, Australia & Lady finger & a & IV \\
\hline PHIL16 & Philippines & Latundan & $\mathrm{e}$ & $\mathrm{V}$ & 23529 & South Johnstone, Australia & Ducasse & a & IV \\
\hline PHIL17 & Philippines & Abaca (Musa textilis) & e & $\mathrm{V}$ & 23604 & Petches Creek, Australia & Lady finger & $\mathrm{a}$ & IV \\
\hline PHIL19 & Philippines & Latundan & $\mathrm{e}$ & $\mathrm{V}$ & M5386 & Mareeba, Australia & Ducasse & $\mathrm{a}$ & IV \\
\hline THAI1-2 & Thailand & Kluai Namwa (ABB) & $\mathrm{f}$ & $\mathrm{V}$ & 23906 & Pimpama, Australia & Lady finger & $\mathrm{a}$ & IV \\
\hline THAI2-1 & Thailand & Kluai Namwa & $\mathrm{f}$ & $\mathrm{V}$ & INDIA1 & India & Mysore (AAB) & $?$ & IV \\
\hline THAI3-1 & Thailand & Kluai Namwa & $\mathrm{f}$ & $\mathrm{V}$ & INDIA2 & India & Mysore & $?$ & IV \\
\hline
\end{tabular}

(continued on the next page)

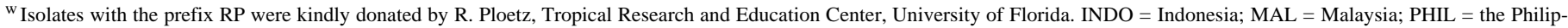
pines; and THAI = Thailand.

${ }^{\mathrm{x}}$ Host genotypes are inter- and intraspecific hybrids of Musa acuminata (A) and M. balbisiana (B). ? = genomic constitution unknown.

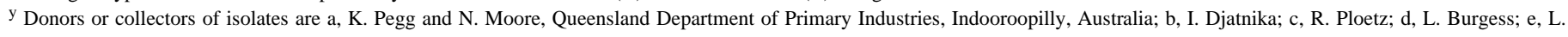
Magnaye; f, N. Singburaudom; g, D. Jones; h, I. Buddenhagen; i, R. Shivas; j, J. C. Bartlett; k, J. Sinurat; 1, H. Stover; m, G. P. Salingay; and n, Y. Doon. ? = donor or collector unknown. ${ }^{\mathrm{z}} \mathrm{DFG}=$ DNA fingerprint group. 
(Table 2). Isolates were classified as 'VCG unknown' either because they had not yet been typed by VCG analysis prior to DNA fingerprinting or they were not compatible with the available set of NitM testers representing the 20 currently recognized VCGs (N. Y. Moore, unpublished data). The isolates that had not been analyzed prior to DNA fingerprinting, but were compatible with the existing VCGs (N. Y. Moore, unpublished data), were initially treated as VCG unknown to test the ability of DNA fingerprinting to determine putative VCG. Isolates were stored as monoconidial cultures that had been grown on sterile moist filter paper that was dried and stored at $4^{\circ} \mathrm{C}$. Each isolate was analyzed at least twice by DNA fingerprinting.
DAF and data analysis. The modified DAF system described by Bentley and Bassam (1) was used with the following minor modifications. For DNA purification, isolates of $F$. oxysporum $\mathrm{f}$. sp. cubense were cultured on carnation leaf agar plates at $25^{\circ} \mathrm{C}$ for 4 to 5 days. These cultures were used to inoculate 250-ml Erlenmeyer flasks containing $200 \mathrm{ml}$ of quarter-strength potato dextrose broth and incubated at room temperature without shaking for no longer than 7 days. The DNA amplification reactions, thermocycling, and electrophoresis conditions are described by Bentley and Bassam (1). Primer sequences used are listed in Table 3. The similarity between different isolates of $F$. oxysporum f. sp. cubense

TABLE 1. (continued from the preceding page)

\begin{tabular}{|c|c|c|c|c|c|c|c|c|c|}
\hline $\begin{array}{l}\text { Accession } \\
\text { number }\end{array}$ & $\begin{array}{c}\text { Geographic } \\
\text { origin }\end{array}$ & $\begin{array}{c}\text { Host origin } \\
\text { and genotype }\end{array}$ & $\begin{array}{l}\text { Donor or } \\
\text { collector }^{\mathrm{y}}\end{array}$ & $\mathrm{DFG}^{\mathrm{z}}$ & $\begin{array}{l}\text { Accession } \\
\text { number }\end{array}$ & $\begin{array}{c}\text { Geographic } \\
\text { origin }\end{array}$ & $\begin{array}{c}\text { Host origin } \\
\text { and genotype }\end{array}$ & $\begin{array}{l}\text { Donor or } \\
\text { collector }^{\mathrm{y}}\end{array}$ & $\mathrm{DFG}^{\mathrm{Z}}$ \\
\hline THAI7-1 & Thailand & Kluai Namwa & $\mathrm{f}$ & IV & MAL18 & Malaysia & Pisang Raja (AAB) & $?$ & III \\
\hline RP42 (1S?) & Bodles, Jamaica & Unknown & $\mathrm{c}$ & IV & MAL20 & Malaysia & Pisang Berangan (AA) & $?$ & III \\
\hline RP43 (STPA3) & Uganda & Pisang awak & $\mathrm{c}$ & IV & MAL32 & Malaysia & Pisang Rastali (AAB) & $\mathrm{h}$ & III \\
\hline RP44 (STNP5) & Zaire & Ney poovan (AB) & c & IV & INDO26 & Indonesia & Pisang Kepok (BBB) & 1 & III \\
\hline VCG 0126 & & & & & INDO30 & Indonesia & Pisang Susu (AAA) & 1 & III \\
\hline PHIL6 & Philippines & Latundan & $\mathrm{e}$ & II & INDO32 & Indonesia & Pisang Berangan & 1 & III \\
\hline PHIL7 & Philippines & Cardaba (BBB) & $\mathrm{e}$ & II & RPJAK1 & Indonesia & Unknown & $\mathrm{c}$ & III \\
\hline INDO33 & Indonesia & Pisang Manurung (ABB) & $\mathrm{h}$ & II & INDO34 & Indonesia & Pisang Berangan & h & III \\
\hline INDO38 & Indonesia & Pisang Rubus (?) & $\mathrm{i}$ & II & INDO52 & Indonesia & Cavendish cv. Grande Naine & $\mathrm{h}, \mathrm{j}$ & III \\
\hline INDO40 & Indonesia & Pisang Manurung & $\mathrm{h}, \mathrm{j}$ & II & INDO56 & Indonesia & Cavendish cv. Valery (AAA) & $\mathrm{h}, \mathrm{m}$ & III \\
\hline INDO41 & Indonesia & Pisang Manurung & $\mathrm{h}, \mathrm{j}$ & II & VCG 01214 & & & & \\
\hline INDO42 & Indonesia & Unknown & $h, j$ & II & RPMW40 & Malawi & Harare & $\mathrm{c}$ & VII \\
\hline INDO43 & Indonesia & Pisang Manurung & $h, j$ & II & VCG 01215 & & & & \\
\hline INDO44 & Indonesia & Pisang Manurung & $\mathrm{h}, \mathrm{j}$ & II & RPCR1-1 & Costa Rica & Gros Michel & $\mathrm{c}$ & I \\
\hline INDO45 & Indonesia & Pisang Manurung & $h, j$ & II & VCG 01216 & & & & \\
\hline INDO57 & Indonesia & Pisang Manurung & $\mathrm{j}, \mathrm{k}$ & II & INDO39 & Indonesia & Pisang Berangan & $\mathrm{h}$ & III \\
\hline INDO58 & Indonesia & Pisang Manurung & $\mathrm{j}, \mathrm{k}$ & II & INDO47 & Indonesia & Cavendish cv. Grande Naine & $\mathrm{h}, \mathrm{j}$ & III \\
\hline INDO59 & Indonesia & Pisang Manurung & $\mathrm{j}, \mathrm{k}$ & II & INDO48 & Indonesia & Pisang Berangan & $\mathrm{h}, \mathrm{j}$ & III \\
\hline INDO60 & Indonesia & Pisang Manurung & $\mathrm{j}, \mathrm{k}$ & II & INDO50 & Indonesia & Cavendish cv. Grande Naine & $\mathrm{h}, \mathrm{j}$ & III \\
\hline INDO63 & Indonesia & Highgate & $\mathrm{j}, \mathrm{k}$ & II & INDO53 & Indonesia & Cavendish cv. Grande Naine & $\mathrm{h}, \mathrm{j}$ & III \\
\hline INDO72 & Indonesia & Pisang Puju (?) & $\mathrm{j}, \mathrm{k}$ & II & MAL1 & Malaysia & Pisang Raja & $?$ & III \\
\hline RPJAK2 & Indonesia & Highgate & c & II & MAL4 & Malaysia & Pisang Raja & $?$ & III \\
\hline RPJAK4 & Indonesia & Highgate & $\mathrm{c}$ & II & MAL5 & Malaysia & Pisang Raja & $?$ & III \\
\hline RP45 (S1) & Honduras & Highgate & $\mathrm{c}$ & II & MAL11 & Malaysia & Pisang Mas & $?$ & III \\
\hline RP46 (STM3) & Honduras & Maqueño (AAB) & $\mathrm{c}$ & II & MAL14 & Malaysia & Pisang Kebatu (ABB) & $?$ & III \\
\hline RP48 (STB2) & Honduras & Highgate & $\mathrm{c}$ & II & MAL21 & Malaysia & Pisang Berangan & $\mathrm{g}$ & III \\
\hline RP49 (4S1) & Honduras & Maqueño & $\mathrm{c}$ & II & MAL22 & Malaysia & Cavendish cv. Grande Naine & $\mathrm{g}$ & III \\
\hline RP50 (5S1) & Honduras & Maqueño & $\mathrm{c}$ & II & MAL26 & Malaysia & Cavendish & $\mathrm{n}$ & III \\
\hline VCG 0128 & & & & & MAL27 & Malaysia & Pisang Rastali & $\mathrm{n}$ & III \\
\hline 22993 & South Johnstone, Australia & Blue Java (ABB) & $\mathrm{a}$ & IV & MAL28 & Malaysia & Unknown & $\mathrm{n}$ & III \\
\hline 22994 & South Johnstone, Australia & Bluggoe & $\mathrm{a}$ & IV & MAL29 & Malaysia & Pisang Berangan & $?$ & III \\
\hline 23909 & Kamerunga, Australia & Bluggoe & $\mathrm{a}$ & IV & MAL31 & Malaysia & Pisang Rastali & $\mathrm{h}$ & III \\
\hline 23996 & Kamerunga, Australia & Bluggoe & $\mathrm{a}$ & IV & MAL34 & Malaysia & Pisang Rastali & $\mathrm{h}$ & III \\
\hline 24235 & Australia & Unknown & a & IV & MAL36 & Malaysia & Cavendish cv. Williams & $\mathrm{h}$ & III \\
\hline 24246 & South Johnstone, Australia & Monthan (ABB) & $\mathrm{a}$ & IV & MAL37 & Malaysia & Cavendish cv. Williams & $\mathrm{h}$ & III \\
\hline 24247 & South Johnstone, Australia & Tuu Gia (AA) & a & IV & MAL38 & Malaysia & Pisang Serandah (AAA) & $\mathrm{h}$ & III \\
\hline 24249 & South Johnstone, Australia & Dwarf Yawa (ABB) & a & IV & MAL39 & Malaysia & Cavendish cv. Grande Naine & $\mathrm{h}$ & III \\
\hline 24250 & South Johnstone, Australia & Kluai Nui Mue Nang (ABB) & a & IV & MAL40 & Malaysia & Cavendish cv. Grande Naine & $\mathrm{h}$ & III \\
\hline 24251 & South Johnstone, Australia & Blue Java & a & IV & MAL41 & Malaysia & Cavendish cv. Grande Naine & $\mathrm{h}$ & III \\
\hline 24253 & South Johnstone, Australia & Tukuru (ABB) & a & IV & VCG 01217 & & & & \\
\hline 24255 & South Johnstone, Australia & Silver Bluggoe (ABB) & a & IV & MAL7 & Malaysia & Pisang Rastali & $?$ & V \\
\hline VCG 0129 & & & & & MAL8 & Malaysia & Pisang Rastali & $?$ & V \\
\hline 8617 & Mooloolah, Australia & Cavendish & $\mathrm{a}$ & $\mathrm{V}$ & MAL23 & Malaysia & Pisang Kebatu & $\mathrm{g}$ & V \\
\hline 24234 & Mooloolah, Australia & Cavendish & $\mathrm{a}$ & V & MAL30 & Malaysia & Pisang Rastali & $\mathrm{h}$ & $\mathrm{V}$ \\
\hline 23509 & Gunalda, Australia & Lady finger & $\mathrm{a}$ & $\mathrm{V}$ & MAL43 & Malaysia & Pisang Rastali & h & V \\
\hline 23510 & Gympie, Australia & Lady finger & a & $\mathrm{V}$ & 23775 & Malaysia & Pisang Rastali & a & $\mathrm{V}$ \\
\hline 23512 & Wappa Dam, Australia & Lady finger & $\mathrm{a}$ & $\mathrm{V}$ & VCG 01218 & & & & \\
\hline 23518 & Kin Kin, Australia & Lady finger & $\mathrm{a}$ & $\mathrm{V}$ & INDO5 & Indonesia & Pisang Siem (ABB) & $\mathrm{b}$ & VI \\
\hline VCG 01210 & & & & & VCG 01219 & & & & \\
\hline RP51 (A1-1) & Florida, United States & Apple & $\mathrm{c}$ & II & INDO25 & Indonesia & Pisang Ambon (AAA) & 1 & II \\
\hline RP52 (A2-1) & Florida, United States & Apple & $\mathrm{c}$ & II & INDO35 & Indonesia & Pisang Raja Sereh (AAB) & $\mathrm{h}$ & II \\
\hline RP53 (GG1) & Florida, United States & Apple & $\mathrm{c}$ & II & INDO36 & Indonesia & Pisang Garing (?) & $\mathrm{h}$ & II \\
\hline RP54 (JC4) & Florida, United States & Apple & $\mathrm{c}$ & II & INDO37 & Indonesia & Pisang Ambon Putih & $\mathrm{h}$ & II \\
\hline VCG 01211 & & & & & VCG 01220 & & & & \\
\hline 23631 & Wamuran, Australia & SH3142 (AA) & $\mathrm{a}$ & I & 24200 & Carnarvon, Western Australia & Cavendish cv. Williams & a & IV \\
\hline RP57 (13721) & $?$ & Unknown & $\mathrm{c}$ & I & 24208 & Carnarvon, Western Australia & Cavendish cv. Williams & a & IV \\
\hline VCG 01212 & & & & & 24218 & Carnarvon, Western Australia & Cavendish cv. Williams & $\mathrm{a}$ & IV \\
\hline RP58 (STNP1) & Tanzania & Ney poovan & c & IV & 24220 & Carnarvon, Western Australia & Cavendish cv. Williams & $\mathrm{a}$ & IV \\
\hline VCG 01213 & & & & & 24211 & Carnarvon, Western Australia & Cavendish cv. Williams & $\mathrm{a}$ & IV \\
\hline MAL15 & Malaysia & Pisang Mas (AA) & $?$ & III & 24219 & Carnarvon, Western Australia & Cavendish cv. Williams & a & IV \\
\hline MAL17 & Malaysia & Pisang Mas & $?$ & III & & & & & \\
\hline
\end{tabular}


was determined using Gel Compar v. 3.1 (1). A total of 483 fragments was scored for the isolates from known VCGs with all 10 primers; 351 were polymorphic (Table 3). To determine the relationships between the VCGs based on all primers, a similarity matrix that rep- resents the average of the values from the 10 similarity matrices was clustered by the unweighted pair group method with arithmetic means (UPGMA) using the SAHN module of NTSYSpc v. 2.0. The goodness-of-fit of the phenogram was determined by

TABLE 2. Isolates of Fusarium oxysporum $\mathrm{f}$. sp. cubense of unknown vegetative compatibility group (VCG) identified by DNA fingerprinting analysis

\begin{tabular}{|c|c|c|c|c|c|}
\hline Accession number ${ }^{v}$ & Geographic origin & Host origin and genotype ${ }^{w}$ & Donor or collector ${ }^{\mathrm{x}}$ & $\mathrm{DFG}^{\mathrm{y}}$ & Genotype $^{z}$ \\
\hline INDO10 & West Java, Indonesia & Pisang Jimbluk (?) & a & V & 01218 \\
\hline INDO16 & West Java, Indonesia & Pisang Ambon Putih (AAA) & a & II & 2 \\
\hline INDO54 & East Java, Indonesia & Pisang Susu (AAB) & $\mathrm{b}, \mathrm{c}$ & II & 3 \\
\hline INDO65 & West Sumatra, Indonesia & Kinalun/Gajah (?) & $\mathrm{d}$ & III & 0121 \\
\hline INDO67 & West Sumatra, Indonesia & Pisang Buai (AAA?) & $\mathrm{d}$ & III & 0121 \\
\hline INDO69 & West Sumatra, Indonesia & Pisang Kepok (BBB) & $\mathrm{d}$ & IV & $0124 / 5$ \\
\hline INDO77 & Sumatra, Indonesia & Cavendish cv. Williams (AAA) & $e, f$ & III & $01213 / 16$ \\
\hline INDO78 & Sumatra, Indonesia & Cavendish (AAA) & $e, f$ & III & $01213 / 16$ \\
\hline INDO79 & Sumatra, Indonesia & Umalag (AAA) & $e, f$ & III & $01213 / 16$ \\
\hline INDO80 & East Java, Indonesia & Pisang Susu & $\mathrm{b}, \mathrm{c}$ & $\mathrm{V}$ & 0123 \\
\hline INDO81 & East Java, Indonesia & Pisang Ambon (AAA) & $\mathrm{b}, \mathrm{c}$ & $\mathrm{I}$ & $0120 / 15$ \\
\hline INDO82 & East Java, Indonesia & Pisang Berangan (AA) & $\mathrm{b}, \mathrm{c}$ & III & $01213 / 16$ \\
\hline INDO83 & East Java, Indonesia & Pisang Ambon Lumut (AAA) & $\mathrm{b}, \mathrm{c}$ & III & $01213 / 16$ \\
\hline INDO84 & East Java, Indonesia & Cavendish cv. Williams & $\mathrm{b}, \mathrm{c}$ & III & $01213 / 16$ \\
\hline INDO85 & East Java, Indonesia & Pisang Ambon Kuning (?) & $\mathrm{b}, \mathrm{c}$ & III & $01213 / 16$ \\
\hline INDO87 & Sulawesi, Indonesia & Pisang Raja Sereh (AAB) & $\mathrm{b}, \mathrm{c}$ & III & $01213 / 16$ \\
\hline INDO88 & Sulawesi, Indonesia & Unknown & $\mathrm{b}, \mathrm{c}$ & II & 0126 \\
\hline INDO89 & Sulawesi, Indonesia & Pisang Puju (?) & $\mathrm{b}, \mathrm{c}$ & II & 0126 \\
\hline INDO90 & Sulawesi, Indonesia & Pisang Puju & $\mathrm{b}, \mathrm{c}$ & II & 0126 \\
\hline INDO91 & Sulawesi, Indonesia & Pisang Ambon & $\mathrm{b}, \mathrm{c}$ & II & 0126 \\
\hline INDO92 & Halmahera, Indonesia & Sangate (AAA?) & $\mathrm{b}, \mathrm{c}$ & II & 0126 \\
\hline INDO93 & Halmahera, Indonesia & Cavendish cv. Grande Naine (AAA) & $\mathrm{b}, \mathrm{c}$ & $\mathrm{I}$ & 0120 \\
\hline INDO94 & Halmahera, Indonesia & Cavendish & $\mathrm{b}, \mathrm{c}$ & III & $01213 / 16$ \\
\hline INDO95 & Halmahera, Indonesia & Mulubebe (?) & $\mathrm{b}, \mathrm{c}$ & III & 0121 \\
\hline INDO96 & Halmahera, Indonesia & Sangate & $\mathrm{b}, \mathrm{c}$ & II & 0126 \\
\hline INDO97 & Halmahera, Indonesia & Cavendish & $\mathrm{b}, \mathrm{c}$ & III & $01213 / 16$ \\
\hline INDO98 & Halmahera, Indonesia & Cavendish & $\mathrm{b}, \mathrm{c}$ & III & $01213 / 16$ \\
\hline INDO99 & Halmahera, Indonesia & Unknown & $b, c$ & II & 0126 \\
\hline INDO100 & East Java, Indonesia & Pisang Putot (?) & $\mathrm{b}, \mathrm{c}$ & III & $01213 / 16$ \\
\hline INDO101 & Sulawesi, Indonesia & Pisang Ambon Lumut & $\mathrm{c}, \mathrm{g}$ & II & 0126 \\
\hline INDO102 & Sulawesi, Indonesia & Pisang Ambon Lumut & $\mathrm{c}, \mathrm{g}$ & II & 0126 \\
\hline THAI1-1 & Petchabun, Thailand & Kluai Namwa (ABB) & $\mathrm{h}$ & $\mathrm{V}$ & 0123 \\
\hline THAI22 & Nong' Khai, Thailand & Kluai Namwa & $\mathrm{i}$ & $\mathrm{V}$ & 01218 \\
\hline THAI23 & Phuket Island, Thailand & Kluai Namwa & $\mathrm{i}$ & IV & $0124 / 5$ \\
\hline THAI24 & Chumporn, Thailand & Kluai Namwa & $\mathrm{i}$ & $\mathrm{V}$ & 01218 \\
\hline THAI25 & Chiang Rai Province, Thailand & Kluai Namwa & $\mathrm{j}$ & $\mathrm{V}$ & 0123 \\
\hline THAI26 & Chiang Mai Province, Thailand & Kluai Namwa & $\mathrm{j}$ & $\mathrm{V}$ & $0124 / 5$ \\
\hline THAI27 & Chiang Mai Province, Thailand & Kluai Namwa & $\mathrm{j}$ & IV & 01220 \\
\hline THAI28 & Chiang Mai Province, Thailand & Kluai Namwa & $\mathrm{j}$ & IV & 01220 \\
\hline THAI29 & Nan Province, Thailand & Kluai Namwa & $\mathrm{j}$ & IV & 0123 \\
\hline THAI30 & Phrae Province, Thailand & Kluai Namwa & $\mathrm{j}$ & $\mathrm{V}$ & 0123 \\
\hline THAI31 & Uttaradit Province, Thailand & Kluai Namwa & $\mathrm{j}$ & $\mathrm{V}$ & 0123 \\
\hline THAI32 & Uttaradit Province, Thailand & Kluai Namwa & $\mathrm{j}$ & $\mathrm{V}$ & 0123 \\
\hline THAI33 & Chiang Rai Province, Thailand & Kluai Namwa & $\mathrm{j}$ & $\mathrm{V}$ & 0123 \\
\hline THAI34 & Chiang Rai Province, Thailand & Kluai Namwa & $\mathrm{j}$ & $\mathrm{V}$ & 0123 \\
\hline THAI35 & Chiang Rai Province, Thailand & Kluai Namwa & $\mathrm{j}$ & $\mathrm{V}$ & 12 \\
\hline THAI36 & Chiang Rai Province, Thailand & Kluai Namwa & $\mathrm{j}$ & $\mathrm{V}$ & 0123 \\
\hline THAI37 & Chiang Rai Province, Thailand & Kluai Namwa & $\mathrm{j}$ & $\mathrm{V}$ & 0123 \\
\hline THAI38 & Chiang Rai Province, Thailand & Kluai Namwa & $\mathrm{j}$ & $\mathrm{V}$ & 0123 \\
\hline THAI39 & Chiang Rai Province, Thailand & Kluai Namwa & $\mathrm{j}$ & IV & $0124 / 5$ \\
\hline MAL4 & Kota Sarang Semut, Malaysia & Pisang awak (AAB) & $?$ & IV & 4 \\
\hline MAL44 & Selangor, Malaysia & Pisang Lilin (AA) & $\mathrm{k}$ & III & $01213 / 16$ \\
\hline MAL45 & Selangor, Malaysia & Pisang Lilin & $\mathrm{k}$ & III & $01213 / 16$ \\
\hline MAL46 & Selangor, Malaysia & Pisang Lilin & $\mathrm{k}$ & III & $01213 / 16$ \\
\hline MAL47 & Selangor, Malaysia & Cavendish cv. Williams & $\mathrm{b}, 1, \mathrm{~m}$ & III & $01213 / 16$ \\
\hline MAL48 & Selangor, Malaysia & Bluggoe (ABB) & $\mathrm{b}, 1, \mathrm{~m}$ & III & $01213 / 16$ \\
\hline MAL49 & Selangor, Malaysia & Gros Michel (AAA) & $\mathrm{b}, 1, \mathrm{~m}$ & III & $01213 / 16$ \\
\hline MAL50 & Selangor, Malaysia & Pisang Mas (AA) & $\mathrm{b}, 1, \mathrm{~m}$ & III & $01213 / 16$ \\
\hline MAL51 & Selangor, Malaysia & Kuda (AA) & $\mathrm{b}, 1, \mathrm{~m}$ & III & $01213 / 16$ \\
\hline MAL52 & Pahang, Malaysia & Unknown (AAB) & $\mathrm{b}, 1, \mathrm{~m}$ & III & $01213 / 16$ \\
\hline MAL53 & Pahang, Malaysia & Unknown (AAB) & $\mathrm{b}, 1, \mathrm{~m}$ & III & $01213 / 16$ \\
\hline
\end{tabular}

${ }^{\mathrm{v}}$ HOND $=$ Honduras; INDO = Indonesia MAL = Malaysia MEX = Mexico $;$ PHIL $=$ the Philippines $;$ THAI $=$ Thailand and VIET $=$ Viet Nam.

${ }^{w}$ Host genotypes are inter- and intraspecific hybrids of Musa acuminata (A) and M. balbisiana (B). ? = genomic constitution unknown.

${ }^{x}$ Donors or collectors of isolates are a, I. Djatnika; b, I. Buddenhagen; c, J. C. Bartlett; d, Jumjunidang; e, Stefanus; f, G. P. Salingay; g, J. Sinurat; h, N. Singburaudom; i, D. Jones; j, S. Kooariyakul; k, S. H. Jamuluddin; 1, N. Moore; m, S. Bentley; n, T. Y. Hock; o, L. Magnaye; p, E. Aguilar; q, S. Medina; r, H. H. Nhi; s, D. T. Thanh; t, J. Stanton; u, K. Pegg; v, R. Caid; and w, J. Duff. ? = donor or collector unknown.

${ }^{y}$ DFG $=$ DNA fingerprint group.

${ }^{z}$ The new genotypes identified in this study were numbered consecutively, whereas genotypes that were identical to an existing VCG were referred to by their VCG code. 
computing a cophenetic value matrix using the $\mathrm{COPH}$ module and comparing this matrix with the SAHN tree matrix using the MXCOMP module. A cophenetic correlation of $r>9.0$ is considered a very good fit.

\section{RESULTS}

Genetic variation within each VCG of $F$. oxysporum f. sp. cubense. Isolates within each VCG generally produced an identi-

TABLE 2. (continued from the preceding page)

\begin{tabular}{|c|c|c|c|c|c|}
\hline Accession number ${ }^{v}$ & Geographic origin & Host origin and genotype ${ }^{w}$ & Donor or collector $^{\mathrm{X}}$ & $\mathrm{DFG}^{\mathrm{y}}$ & Genotype $^{z}$ \\
\hline MAL54 & Pahang, Malaysia & Unknown (AAB) & $\mathrm{b}, 1, \mathrm{~m}$ & III & $01213 / 16$ \\
\hline MAL55 & Pahang, Malaysia & Pisang awak & $\mathrm{b}, 1, \mathrm{~m}$ & $\mathrm{~V}$ & 0123 \\
\hline MAL57 & Negeri Sembilan, Malaysia & Pisang Rastali (AAB) & $\mathrm{b}, 1, \mathrm{~m}$ & $\mathrm{~V}$ & 01217 \\
\hline MAL58 & Negeri Sembilan, Malaysia & Pisang Rastali & $\mathrm{b}, \mathrm{l}, \mathrm{m}$ & V & 01217 \\
\hline MAL59 & Negeri Sembilan, Malaysia & Cavendish cv. Williams & $\mathrm{b}, 1, \mathrm{~m}$ & III & $01213 / 16$ \\
\hline MAL60 & Negeri Sembilan, Malaysia & Cavendish cv. Williams & $\mathrm{b}, 1, \mathrm{~m}$ & III & $01213 / 16$ \\
\hline MAL61 & Negeri Sembilan, Malaysia & Pisang Rastali & $\mathrm{b}, 1, \mathrm{~m}$ & III & $01213 / 16$ \\
\hline MAL62 & Negeri Sembilan, Malaysia & Pisang Rastali & $\mathrm{b}, 1, \mathrm{~m}$ & V & 01217 \\
\hline MAL63 & Negeri Sembilan, Malaysia & Pisang Rastali & $\mathrm{b}, \mathrm{l}, \mathrm{m}$ & $\mathrm{V}$ & 01217 \\
\hline MAL64 & Negeri Sembilan, Malaysia & Pisang Rastali & $\mathrm{b}, \mathrm{l}, \mathrm{m}$ & V & 01217 \\
\hline MAL65 & Negeri Sembilan, Malaysia & Musa acuminata subsp. malaccensis & $\mathrm{b}, \mathrm{l}, \mathrm{m}$ & $\mathrm{V}$ & 5 \\
\hline MAL66 & Negeri Sembilan, Malaysia & Musa acuminata subsp. malaccensis & $\mathrm{b}, \mathrm{l}, \mathrm{m}$ & V & 5 \\
\hline MAL67 & Negeri Sembilan, Malaysia & Musa acuminata subsp. malaccensis & $\mathrm{b}, 1, \mathrm{~m}$ & $\mathrm{~V}$ & 5 \\
\hline MAL68 & Negeri Sembilan, Malaysia & Pisang Kapas (?) & $\mathrm{b}, 1, \mathrm{~m}$ & III & $01213 / 16$ \\
\hline MAL69 & Near Melaka, Malaysia & Unknown (AAB) & $\mathrm{b}, 1, \mathrm{~m}$ & III & $01213 / 16$ \\
\hline MAL70 & Johor, Malaysia & Cavendish cv. Grande Naine & $\mathrm{b}, \mathrm{l}, \mathrm{m}$ & III & $01213 / 16$ \\
\hline MAL71 & Johor, Malaysia & Cavendish cv. Grande Naine & $\mathrm{b}, \mathrm{l}, \mathrm{m}$ & III & $01213 / 16$ \\
\hline MAL72 & Johor, Malaysia & Cavendish cv. Grande Naine & $\mathrm{b}, \mathrm{l}, \mathrm{m}$ & III & $01213 / 16$ \\
\hline MAL73 & Johor, Malaysia & Pisang Rastali & $\mathrm{b}, 1, \mathrm{~m}$ & V & 0123 \\
\hline MAL74 & Johor, Malaysia & Cavendish cv. Grande Naine & $\mathrm{b}, 1, \mathrm{~m}$ & III & 0121 \\
\hline MAL75 & Johor, Malaysia & Cavendish cv. Grande Naine & $\mathrm{b}, \mathrm{l}, \mathrm{m}$ & III & 0121 \\
\hline MAL76 & Johor, Malaysia & Cavendish cv. Grande Naine & $\mathrm{b}, \mathrm{l}, \mathrm{m}$ & III & 0121 \\
\hline MAL77 & Johor, Malaysia & Cavendish cv. Grande Naine & $\mathrm{b}, \mathrm{l}, \mathrm{m}$ & III & 0121 \\
\hline MAL78 & Johor, Malaysia & Cavendish cv. Grande Naine & $\mathrm{b}, \mathrm{l}, \mathrm{m}$ & III & $01213 / 16$ \\
\hline MAL80 & Johor, Malaysia & Cavendish cv. Grande Naine & $\mathrm{b}, \mathrm{l}, \mathrm{m}$ & III & $01213 / 16$ \\
\hline MAL81 & Johor, Malaysia & Cavendish cv. Grande Naine & $\mathrm{b}, \mathrm{l}, \mathrm{m}$ & III & $01213 / 16$ \\
\hline MAL82 & Johor, Malaysia & Cavendish cv. Grande Naine & $\mathrm{b}, \mathrm{l}, \mathrm{m}$ & III & $01213 / 16$ \\
\hline MAL83 & Johor, Malaysia & Cavendish & $\mathrm{n}$ & III & $01213 / 16$ \\
\hline PHIL1 & Mindanao, Philippines & Latundan (AAB) & o & II & 7 \\
\hline PHIL4 & Mindanao, Philippines & Latundan & o & IV & 11 \\
\hline PHIL18 & Luzon, Philippines & Latundan & o & II & 8 \\
\hline PHIL22 & Luzon, Philippines & Katali (ABB) & o & IV & 11 \\
\hline PHIL23 & Luzon, Philippines & Siusok (ABB) & o & IV & 11 \\
\hline PHIL24 & Luzon, Philippines & Latundan & $\mathrm{p}, \mathrm{q}$ & IV & 9 \\
\hline PHIL26 & Luzon, Philippines & Latundan & $\mathrm{p}, \mathrm{q}$ & IV & 10 \\
\hline INDIA7 & India & Poovan (AAB) & $?$ & IV & 01220 \\
\hline VIET1 & So'n La Province, Viet Nam & Chuôi ngôp cao (?) & $\mathrm{r}, \mathrm{s}, \mathrm{t}$ & $\mathrm{V}$ & 13 \\
\hline VIET3 & Tu Liem, Hanôi, Viet Nam & Chuôi xiem (ABB) & $\mathrm{b}, 1, \mathrm{u}$ & IV & 14 \\
\hline VIET4 & Vinh Phú Province, Viet Nam & Chuôi xiem & $\mathrm{b}, \mathrm{l}, \mathrm{u}$ & IV & 14 \\
\hline VIET5 & Vinh Phú Province, Viet Nam & Chuôi xiem & $\mathrm{b}, \mathrm{l}, \mathrm{u}$ & IV & 14 \\
\hline VIET6 & Vinh Phú Province, Viet Nam & Chuôi xiem & $\mathrm{b}, \mathrm{l}, \mathrm{u}$ & IV & 14 \\
\hline VIET7 & Vinh Phú Province, Viet Nam & Chuôi xiem & $\mathrm{b}, \mathrm{l}, \mathrm{u}$ & IV & 14 \\
\hline VIET8 & Thúa Thiên Huê Province, Viet Nam & Chuôi xiem & $\mathrm{b}, \mathrm{l}, \mathrm{u}$ & V & 12 \\
\hline VIET9 & Thúa Thiên Huê Province, Viet Nam & Chuôi xiem & $\mathrm{b}, \mathrm{l}, \mathrm{u}$ & V & 12 \\
\hline VIET10 & Thúa Thiên Huê Province, Viet Nam & Chuôi xiem & $\mathrm{b}, \mathrm{l}, \mathrm{u}$ & V & 12 \\
\hline VIET11 & Thúa Thiên Huê Province, Viet Nam & Chuôi xiem & $\mathrm{b}, \mathrm{l}, \mathrm{u}$ & $\mathrm{V}$ & 12 \\
\hline VIET12 & Thúa Thiên Huê Province, Viet Nam & Chuôi xiem & $\mathrm{b}, 1, \mathrm{u}$ & $\mathrm{V}$ & 12 \\
\hline VIET13 & Thúa Thiên Huê Province, Viet Nam & Chuôi xiem & $1, \mathrm{u}$ & V & 12 \\
\hline VIET14 & Tiên Giang Province, Viet Nam & Chuôi xiem & $\mathrm{u}$ & IV & $0124 / 5$ \\
\hline VIET15 & Tiên Giang Province, Viet Nam & Chuôi xiem & $\mathrm{b}$ & V & 12 \\
\hline VIET16 & Tiên Giang Province, Viet Nam & Chuôi xiem & $\mathrm{b}, 1$ & $\mathrm{~V}$ & 12 \\
\hline VIET17 & Cân Tho Province, Viet Nam & Chuôi xiem & $\mathrm{b}, \mathrm{l}, \mathrm{u}$ & IV & $0124 / 5$ \\
\hline VIET18 & Cân Tho Province, Viet Nam & Chuôi xiem & $\mathrm{b}, \mathrm{l}, \mathrm{u}$ & IV & $0124 / 5$ \\
\hline VIET19 & Cân Tho Province, Viet Nam & Chuôi xiem & $\mathrm{b}, 1, \mathrm{u}$ & $\mathrm{V}$ & 12 \\
\hline VIET20 & Vinh Long Province, Viet Nam & Chuôi xiem & $\mathrm{b}, \mathrm{l}, \mathrm{u}$ & IV & $0124 / 5$ \\
\hline VIET21 & Vinh Long Province, Viet Nam & Chuôi xiem & $\mathrm{b}, \mathrm{l}, \mathrm{u}$ & IV & $0124 / 5$ \\
\hline VIET22 & Tiên Giang Province, Viet Nam & Chuôi xiem & $\mathrm{b}, \mathrm{l}, \mathrm{u}$ & V & 12 \\
\hline MEX1 & Nayarit, Mexico & Silk (Manzano) (AAB) & $\mathrm{b}$ & VIII & 6 \\
\hline MEX2 & Nayarit, Mexico & Silk (AAB) & $\mathrm{b}$ & VIII & 6 \\
\hline MEX3 & Nayarit, Mexico & Silk & $\mathrm{b}$ & VIII & 6 \\
\hline MEX4 & Nayarit, Mexico & Silk & $\mathrm{b}$ & VIII & 6 \\
\hline MEX5 & Nayarit, Mexico & Silk & $\mathrm{b}$ & VIII & 6 \\
\hline MEX6 & Nayarit, Mexico & Silk & $\mathrm{b}$ & VIII & 6 \\
\hline MEX7 & Nayarit, Mexico & Silk & $\mathrm{b}$ & VIII & 6 \\
\hline HOND1 & La Esparanza, Peña, Honduras & Gros Michel & $\mathrm{v}$ & IV & $0124 / 5$ \\
\hline HOND2 & La Esparanza, Peña, Honduras & Gros Michel & $\mathrm{v}$ & IV & $0124 / 5$ \\
\hline HOND3 & La Esparanza, Peña, Honduras & Gros Michel & $\mathrm{v}$ & IV & $0124 / 5$ \\
\hline HOND4 & El Progresso, Honduras & Chato (ABB) & $\mathrm{V}$ & IV & $0124 / 5$ \\
\hline HOND5 & El Progresso, Honduras & Chato & $\mathrm{v}$ & IV & $0124 / 5$ \\
\hline 24405 & Darwin, Australia & Heliconia chartacea & $\mathrm{w}$ & IX & 1 \\
\hline 24406 & Darwin, Australia & Heliconia chartacea & $\mathrm{w}$ & IX & 1 \\
\hline
\end{tabular}


cal DNA fingerprint and were closely related, regardless of geographic origin or host source. The genetic similarity between isolates within each VCG was determined based on primer ILOE (Table 4). All isolates within each of the VCGs 0121, 0122, 0123, $0128,0129,01210,01213,01216,01217,01219$, and 01220 produced an identical DNA fingerprint pattern and, therefore, had a genetic similarity of $100 \%$ (Table 4). All isolates within VCG 0120 produced an identical DNA fingerprint, except for isolates RP1, RP2, and 23607 (Fig. 1). These three isolates all produced one DNA fragment at a reduced intensity compared with the other isolates in VCG 0120 , and isolate 23607 also differed by the intensity of one other fragment. The average genetic similarity among isolates within VCG 0120 was 99\% (Table 4). Isolates within VCG 0126 were very similar to each other, except that isolates PHIL7 and INDO72 were missing one fragment that was present in the DNA fingerprints of the other isolates within this VCG. The two isolates examined within VCG 01211 differed by one fragment. The genetic similarity within the VCGs $01212,01214,01215$, and 01218 could not be determined, as only one isolate was examined from each of these VCGs. Among isolates in VCGs 0124, 0125, 0128, 01212, and 01220 , five genotypes were identified that did not always correlate with the VCG. Within this group, some isolates in the same VCG produced different DNA fingerprints, and some isolates belonging in different VCGs produced identical DNA fingerprints. The DNA fingerprints of isolates in these VCGs differed by the

TABLE 3. Primer sequences and number of polymorphic fragments generated

\begin{tabular}{lccc}
\hline Primer & Sequence 5' $^{\prime}$ to 3' & $\begin{array}{c}\text { Total number of } \\
\text { fragments scored }\end{array}$ & $\begin{array}{c}\text { Number of } \\
\text { polymorphic fragments }\end{array}$ \\
\hline DINQ & CTG GCC CA & 51 & 41 \\
DJDH & ACC AGC CA & 48 & 31 \\
EHKJ & GCT CAC GA & 47 & 29 \\
HIRH & ACG TCC AC & 50 & 33 \\
ILOE & GAT GAG CC & 54 & 41 \\
IMBE & GAA ACG CC & 49 & 32 \\
IMBR & GTA ACG CC & 49 & 38 \\
NRKI & CCT CGT GG & 46 & 40 \\
NROI & CCT GGT GG & 48 & 35 \\
RKMI & CCC GTC GT & 41 & 31 \\
Total & & 483 & 351 \\
\hline
\end{tabular}

TABLE 4. Genetic similarity among isolates within each vegetative compatibility group (VCG) of Fusarium oxysporum f. sp. cubense based on DNA fingerprinting analysis with primer ILOE

\begin{tabular}{lcc}
\hline VCG & Number of isolates analyzed & Average genetic similarity $(\%)$ \\
\hline 0120 & 26 & 99 \\
0121 & 7 & 100 \\
0122 & 7 & 100 \\
0123 & 21 & 100 \\
0124 & 19 & 96 \\
0125 & 18 & 96 \\
0126 & 23 & 99 \\
0128 & 12 & 100 \\
0129 & 6 & 100 \\
01210 & 4 & 100 \\
01211 & 2 & 98 \\
01212 & 1 & $\ldots$ \\
01213 & 12 & 100 \\
01214 & 1 & $\ldots$ \\
01215 & 1 & $\ldots$ \\
01216 & 24 & 100 \\
01217 & 6 & 100 \\
01218 & 1 & $\ldots$ \\
01219 & 4 & 100 \\
01220 & 6 & 100 \\
\hline
\end{tabular}

$\mathrm{y}$ The value given is the average of the values determined for all isolates within a VCG based on the Jaccard similarity coefficient using Gel Compar analysis.

$\mathrm{z}$ The genetic similarity could not be determined as only one isolate was examined from each of these VCGs. presence or absence of one or two fragments, and the genetic similarity among isolates in these VCGs ranged from 96 to $100 \%$.

Genetic variation between different VCGs of $\boldsymbol{F}$. oxysporum f. sp. cubense. As there was minimal variation among isolates within each VCG, an isolate was selected to represent each VCG, and the relationships between the VCGs were determined by comparing these representative isolates using 10 different arbitrary primers (Fig. 2). Each of the primers grouped the isolates similarly based on the respective DNA fingerprint patterns they generated. Although each primer produced similar results, it was possible to differentiate closely related VCGs more easily with some primers than with others. For each of the 10 primers, a similarity matrix was generated based on the Jaccard coefficient using Gel Compar. To determine the relationships between the VCGs based on all primers, a similarity matrix that represented the average of the values from the 10 similarity matrices (Table 5) was clustered by the UPGMA method using the SAHN module of NTSYSpc v. 2.0 (Fig. 3). The obtained cophenetic correlation value of $r=0.99$ indicated that the UPGMA cluster analysis was statistically significant.

The DNA fingerprint patterns generated by most primers revealed a distinct genotype for each different VCG, except for VCGs 01213 and 01216, which produced an identical DNA fingerprint pattern with all primers, i.e., 19 genotypes were differentiated among the 20 reported VCGs of $F$. oxysporum $\mathrm{f}$. sp. cubense. In most instances, each VCG could be distinguished by the presence or absence of more than one fragment. Some VCGs, however, often produced an identical genotype with the majority of primers including VCGs 0120 and 01215, VCGs 0124 and 0125, and VCGs 0129 and 01211.

Identification of isolates of unknown VCG. The isolates of unknown VCG were from Australia, Honduras, India, Indonesia, Malaysia, Mexico, the Philippines, Thailand, and Viet Nam (Table 2). For all isolates that belonged to an existing VCG, the putative VCG determined by DNA fingerprinting agreed with the actual VCG designation (Table 2). Among isolates of unknown VCG, several produced a DNA fingerprint characteristic of an existing VCG, but the isolates were not compatible with that VCG based on tests using the currently available NitM testers. The difference that determines vegetative incompatibility may be only minor, and further screening with more primers may differentiate these iso-

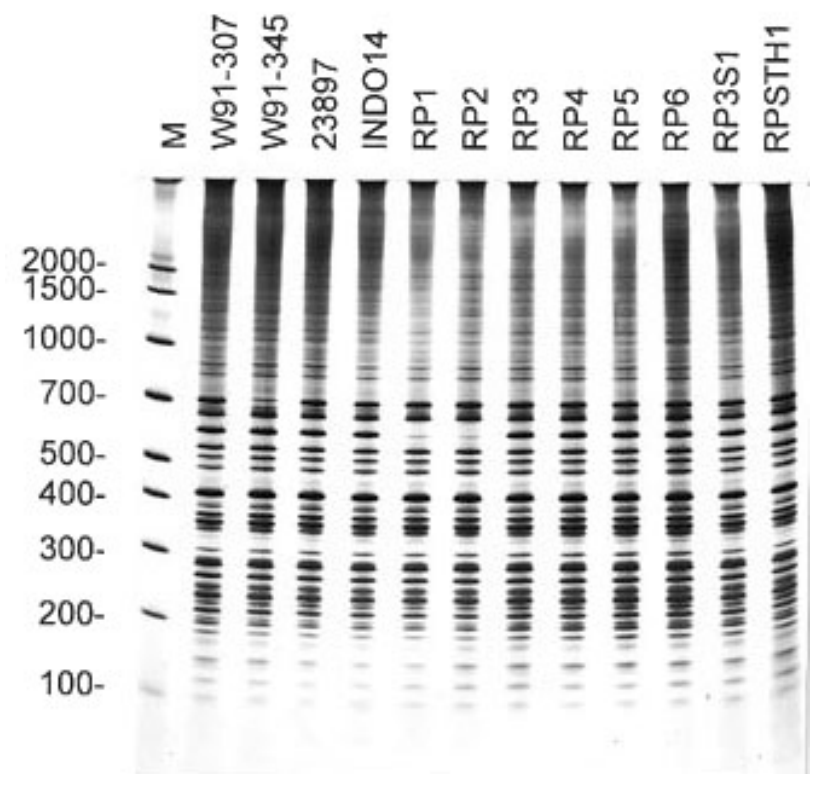

Fig. 1. Genetic variation within vegetative compatibility group (VCG) 0120 of Fusarium oxysporum f. sp. cubense. DNA fingerprints were generated using primer ILOE. The isolate accession numbers are indicated at the top of the figure. The sizes in base pairs of the molecular weight marker are indicated to the left of the figure. 
lates or further vegetative compatibility testing with additional NitM testers may confirm the putative VCG classification. Among the isolates that were not compatible with any of the 20 currently recognized VCGs, 14 new genotypes were identified (Tables 2 and 6). These new genotypes were numbered consecutively (1 to 14), whereas genotypes that represented an existing VCG were referred to by their VCG code.

Clonal lineages within $\boldsymbol{F}$. oxysporum f. sp. cubense. Comparison of the DNA fingerprints, both visually and by phenetic analysis, subdivided isolates of $F$. oxysporum $\mathrm{f}$. sp. cubense into nine clonal lineages (Table 7). The lineages within $F$. oxysporum f. sp. cubense were referred to as DNA fingerprint groups (DFGs). Five of these lineages each contained numerous closely related VCGs and genotypes, and the remaining four lineages each contained only a single VCG or genotype. The genetic similarity of the VCGs and genotypes within each lineage ranged from 80 to $100 \%$. The similarity of the VCGs within each lineage was also evidenced by the presence of VCG cross-compatible isolates within some lineages. There were no VCGs common to more than one lineage.

\section{DISCUSSION}

Relationships between DNA fingerprints and VCGs. In general, there was little or no genetic variation among isolates within each VCG of $F$. oxysporum f. sp. cubense, irrespective of host or geographic origin. Only one DNA fingerprint pattern that represented a unique genotype was distinguished for each VCG, except for VCGs 0120, 0126, and 01211. These VCGs each contained one or two isolates that differed by the intensity or absence of a single DNA fragment. Because the DNA fingerprints were generally VCG specific, it was possible to quickly determine a putative VCG classification for isolates prior to vegetative compatibility analysis. In

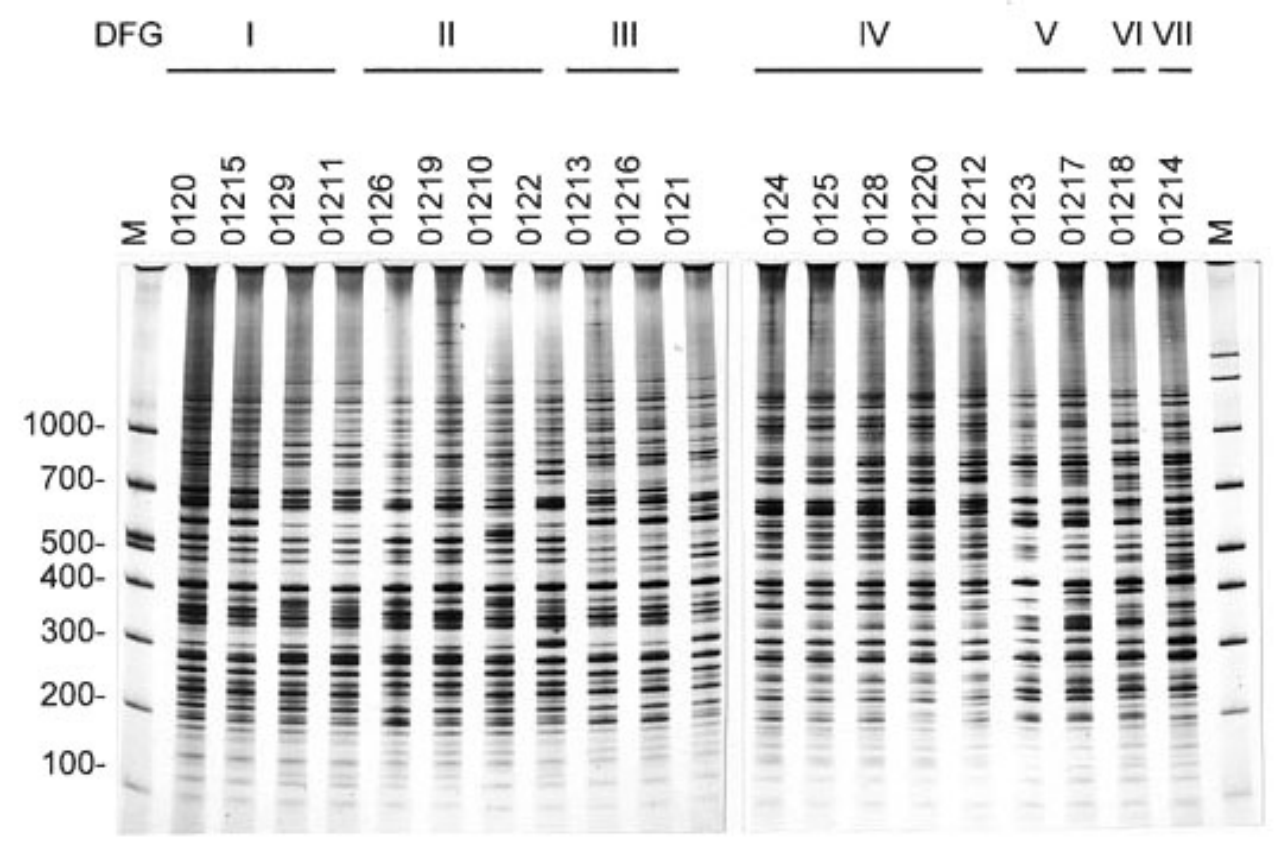

Fig. 2. Genetic variation between different vegetative compatibility groups (VCGs) of Fusarium oxysporum f. sp. cubense. DNA fingerprints were generated using primer ILOE. The VCG codes and DNA fingerprint group (DFG) numbers are indicated at the top of the figure. The sizes in base pairs of the molecular weight marker are indicated to the left of the figure.

TABLE 5. Genetic similarity (\%) between different vegetative compatibility groups (VCGs) of Fusarium oxysporum f. sp. cubense $^{\mathrm{z}}$

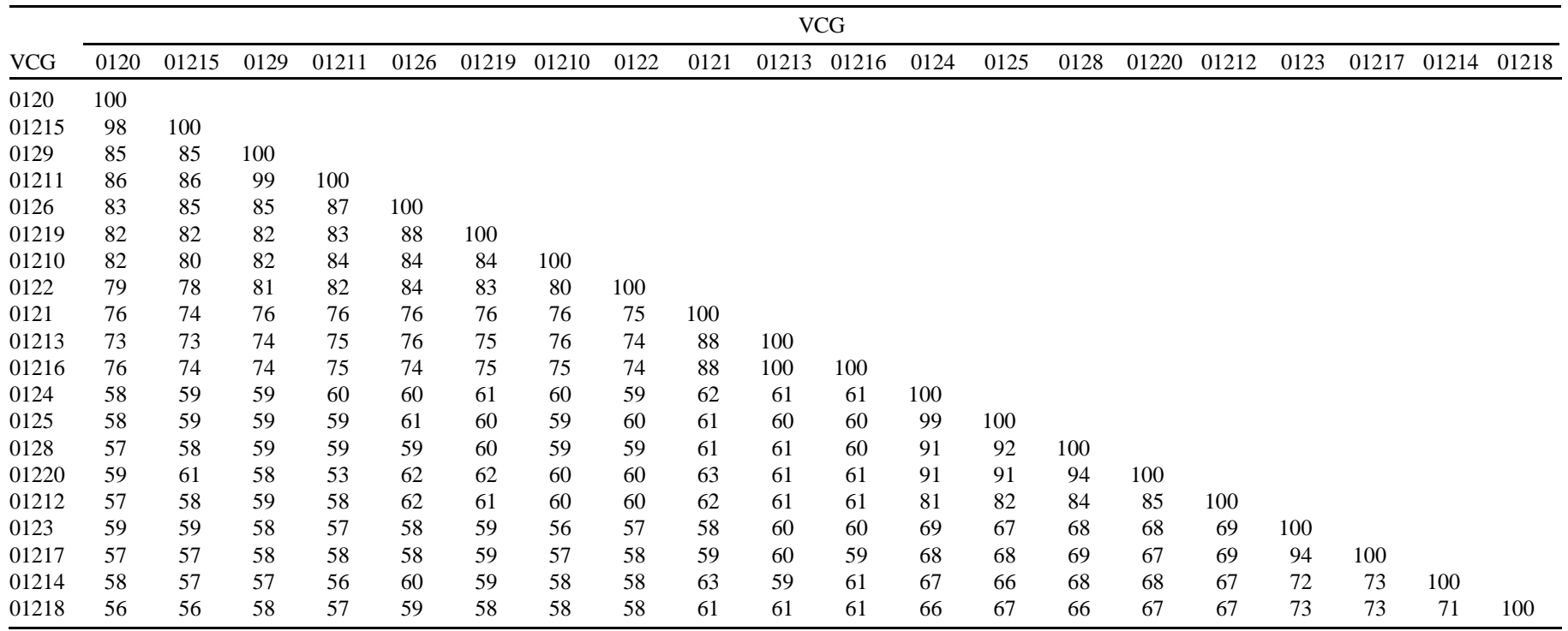

${ }^{\mathrm{z}}$ Each value is the average of the values determined for 10 different primers using the Jaccard similarity coefficient. 
this study, there was near complete correlation between the putative VCG classification determined by DNA fingerprinting and the actual VCG designation. The exception was that several isolates of unknown VCG produced a DNA fingerprint characteristic of an existing VCG but were not compatible with that VCG based on tests using the currently available NitM testers. Further vegetative compatibility testing of these isolates with additional NitM testers has since confirmed the putative VCG classification (N. Y. Moore, unpublished data). DNA fingerprinting also enabled characterization of isolates that were not compatible with any of the existing VCGs. The identification of genetically similar isolates of unknown VCG may facilitate the establishment of new VCGs, as genetically similar isolates are more likely to belong in the same VCG.

Although vegetative compatibility is a useful means of grouping genetically similar isolates, it does not provide any indication of the genetic relatedness between incompatible isolates, such as those from different VCGs and formae speciales, and heterokaryon self-incompatible isolates. A mutation at a single vic locus could result in closely related isolates being vegetatively incompatible and, thus, clonally related isolates may occur in different VCGs. Using DNA fingerprinting analysis, we have determined the genetic variation among isolates in different VCGs of $F$. oxysporum $\mathrm{f}$. sp. cubense including many isolates of unknown VCG. Several different VCGs generated identical DNA fingerprints with the majority of primers including VCGs 0120 and 01215 , VCGs 0124 and 0125, VCGs 0129 and 01211, and VCGs 01213 and 01216. These pairs of VCGs have also been shown to include "bridging" or crosscompatible isolates (N. Y. Moore, unpublished data). The bridging isolates are capable of forming heterokaryons with NitM testers representing both VCGs. VCGs 01213 and 01216 produced identical DNA fingerprint patterns with all the arbitrary primers tested and were considered to be the same genotype. This genotype is significant in that it is capable of severely damaging Cavendish cultivars in the tropics (tropical race 4).

The VCGs and genotypes in DFG IV (VCGs 0124, 0125, 0128, 01212 , and 01220; genotypes $4,9,10,11$, and 14) were also closely related based on their DNA fingerprint patterns. Within this lineage, some isolates in different VCGs produced identical DNA fingerprint, and some isolates in the same VCG produced different DNA fingerprints. The genotypes identified within this lineage correlated with geographic origin rather than VCG or host origin. For example, the isolate in VCG 0124 from Thailand was identical to isolates in VCG 0125 from Thailand, but was different from isolates in VCG 0124 from other countries. Similarly, isolates from the Vinh Phú Province in North Viet Nam belonging in VCGs 0124/5 and 0125 were identical to each other, but distinct from VCG 0124/5 isolates from the Tiên Giang, Cân Tho, and Vinh Long Provinces in

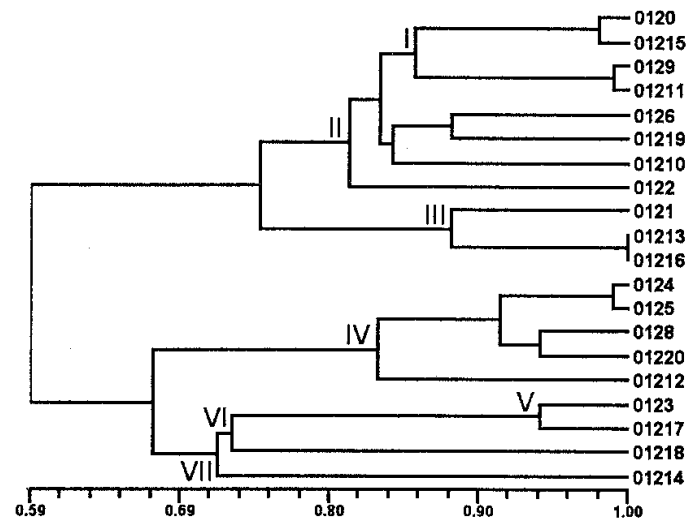

Fig. 3. Genetic similarity between the reported vegetative compatibility groups (VCGs) of Fusarium oxysporum f. sp. cubense. Phenogram represents UPGMA cluster analysis of the average of the similarity values determined for 10 arbitrary primers using the Jaccard similarity coefficient. DNA fingerprint groups (DFGs) identified in this study are indicated on each branch of the phenogram.
South Viet Nam. Based on the similarity of the DNA fingerprint patterns of the genotypes within DFG IV, more rigorous VCG testing with more NitM testers may indicate that other genotypes within this lineage are also cross-compatible.

Clonal lineages within $\boldsymbol{F}$. oxysporum f. sp. cubense. We have identified nine major lineages within $F$. oxysporum f. sp. cubense. The genetic similarity of the VCGs and genotypes within each lineage ranged from 80 to $100 \%$. Within each lineage of $F$. oxysporum f. sp. cubense, the DNA fingerprint pattern of each genotype differed by only one or a few fragments. The similarity of the DNA fingerprint patterns suggests that the genotypes of $F$. oxysporum f. sp. cubense have evolved by mutation within each clonal lineage. The occurrence of VCG cross-compatible isolates within several lineages and the absence of VCGs and genotypes common to different lineages are further evidence that each lineage is clonally derived. Furthermore, the lineages within $F$. oxysporum f. sp. cubense were as different from each other as they were from other formae speciales of $F$. oxysporum (S. Bentley, unpublished data). These results suggest that $F$. oxysporum f. sp. cubense is polyphyletic, which is in agreement with previous studies by Koenig et al. (10) and O'Donnell et al. (16) based on RFLP analysis and DNA sequencing of nuclear and mitochondrial genes, respectively.

The largest lineage (DFG IV) contained 10 different genotypes of worldwide distribution (Fig. 4). The second largest lineage (DFG II) consisted of eight genotypes, all of which originated from Indonesia and the Philippines, except for an isolated population from Florida (VCG 01210). Four of the lineages of $F$. oxysporum f. sp. cubense each contained a single VCG or genotype and were of limited geographic origin. DFG VI was represented by VCG 01218, and although in this study only one isolate was examined, further analysis of more isolates has confirmed the genetic relationship of this VCG to the other VCGs of $F$. oxysporum f. sp. cubense (S. Bentley, unpublished data). DFG VII was represented

TABLE 6. Geographical distribution of genotypes of Fusarium oxysporum f. sp. cubense

\begin{tabular}{|c|c|c|c|c|}
\hline Country & $\begin{array}{l}\text { No. of } \\
\text { isolates } \\
\text { of FOC }\end{array}$ & $\begin{array}{c}\text { Genotypes identified } \\
\text { by DNA fingerprint pattern }{ }^{z}\end{array}$ & $\begin{array}{l}\text { Total no. of } \\
\text { genotypes }\end{array}$ & $\begin{array}{l}\text { Lineages } \\
\text { represented }\end{array}$ \\
\hline Australia & 62 & $\begin{array}{l}0120,0124,0125,0128,0129,01211 \\
01220,1(24405,24406)\end{array}$ & 8 & I, IV, IX \\
\hline Brazil & 1 & 0124 & 1 & IV \\
\hline Canary Islands & 2 & 0120 & 1 & I \\
\hline Costa Rica & 3 & 0120,01215 & 2 & I \\
\hline Honduras & 14 & $0120,0124,0124 / 5,0125,0126$ & 3 & I, II, IV \\
\hline India & 3 & 0125 & 2 & IV \\
\hline Indonesia & 65 & $\begin{array}{l}\text { 0120, 0121, 0124/5, 0126, 01213/16, } \\
\text { 01218, 01219, } 2 \text { (INDO16), } 3 \text { (INDO54) }\end{array}$ & 10 & $\begin{array}{l}\text { I, II, III, IV, } \\
\text { V, VI }\end{array}$ \\
\hline Irian Jaya & 1 & 0126 & 1 & II \\
\hline Jamaica & 3 & $0120,0124,0125$ & 3 & I, IV \\
\hline Malawi & 9 & $0124,0124 / 5,01214$ & 3 & I, V, VII \\
\hline Malaysia & 71 & $\begin{array}{l}\text { 0121, 0123, 01213/16, 01217, } 4 \\
\text { (MAL4), } 5 \text { (MAL65-67) }\end{array}$ & 6 & III, IV, V \\
\hline Mexico & 7 & 6 (MEX1-7) & 1 & VIII \\
\hline Nicaragua & 1 & 0124 & 1 & IV \\
\hline Philippines & 26 & $\begin{array}{l}\text { 0122, 0123, 0126, } 7 \text { (PHIL1), } 8 \\
\text { (PHIL18), 9 (PHIL24), } 10 \text { (PHIL26), } \\
11 \text { (PHIL4, 22, 23) }\end{array}$ & 8 & II, III, IV, V \\
\hline South Africa & 3 & 0120 & 1 & I \\
\hline Taiwan & 9 & 0121,0123 & 2 & III, V \\
\hline Tanzania & 2 & 0124,01212 & 2 & IV \\
\hline Thailand & 30 & $\begin{array}{l}0123,0124,0124 / 5,0125,01218, \\
01220,12 \text { (THAI35) }\end{array}$ & 7 & IV, V, VI \\
\hline Uganda & 1 & 0125 & 1 & IV \\
\hline United States & 6 & $0124,0124 / 5,01210$ & 3 & II, IV \\
\hline Viet Nam & 21 & $\begin{array}{l}\text { 0124/5, } 12 \text { (VIET8-13, 15, 16, 19, 22), } \\
13 \text { (VIET1), } 14 \text { (VIET3-7) }\end{array}$ & 4 & IV, V \\
\hline Zaire & 1 & 0125 & 1 & IV \\
\hline Total & 340 & 19 & 33 & 9 \\
\hline
\end{tabular}

${ }^{\mathrm{z}}$ The new genotypes identified in this study were numbered consecutively; whereas genotypes that represented an existing VCG were referred to by their VCG code. The accession numbers of isolates that represent each new genotype are given in parenthesis. 
by VCG 01214 . Although only a single isolate was available for analysis in this study, Koenig et al. (10) also found this genotype to be genetically distinct from other isolates of $F$. oxysporum $\mathrm{f}$. sp. cubense. Isolates within VCG 01214 are from Malawi and are atypical in that chlamydospores are not produced (10). DFG VIII was represented by one of the new genotypes (genotype 6) that was found attacking the Manzano ('Silk', AAB) cultivar in Nayarit, Mexico, and DFG IX included the race 3 isolates of $F$. oxysporum f. sp. cubense (genotype 1) from a Heliconia species in Australia (Fig. 5).

Previous studies of diversity within $F$. oxysporum f. sp. cubense have divided isolates into two major groups based on volatile analysis $(13,17)$, pectic enzyme analysis (18), electrophoretic karyotyping (4), DNA fingerprinting (1,2,3), RFLP analysis (10), and DNA sequence analysis of nuclear and mitochondrial genes (16).

TABLE 7. Clonal lineages of Fusarium oxysporum f. sp. cubense determined by DNA fingerprinting analysis

\begin{tabular}{ll}
\hline DNA fingerprint group & \multicolumn{1}{c}{ Genotypes $^{\mathrm{z}}$} \\
\hline I & $0120,0129,01211,01215$ \\
II & $0122,0126,01210,01219,2,3,7,8$ \\
III & $0121,01213,01216$ \\
IV & $0124,0125,0128,01212,01220,4,9,10,11,14$ \\
V & $0123,01217,5,12,13$ \\
VI & 01218 \\
VII & 01214 \\
VIII & 6 \\
IX & 1
\end{tabular}

${ }^{z}$ The new genotypes identified in this study were numbered consecutively; whereas genotypes that represented an existing VCG were referred to by their VCG code.

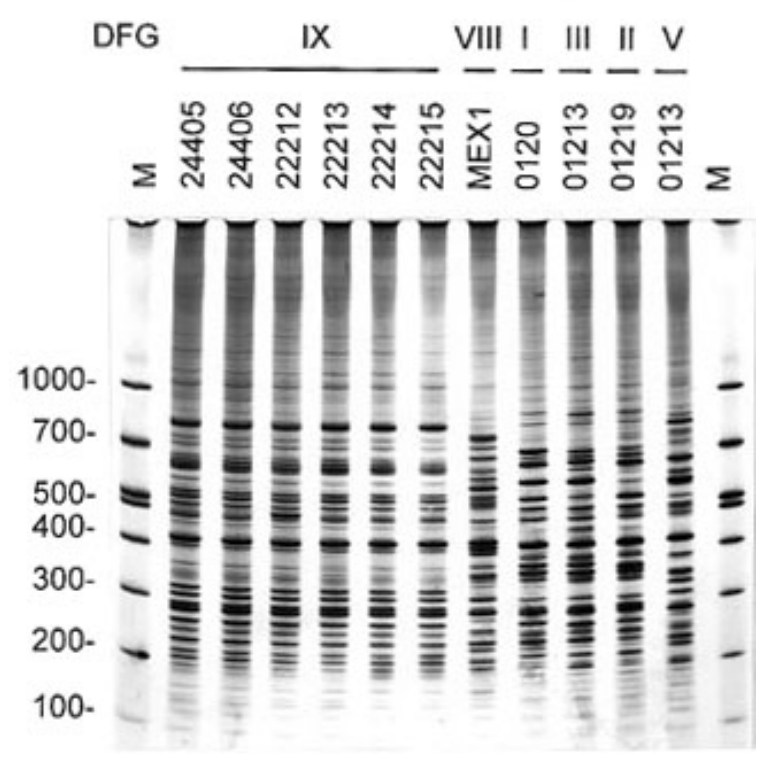

Fig. 5. Comparison of race 3 isolates of Fusarium oxysporum f. sp. cubense from Heliconia species to isolates from banana from Mexico and some vegetative compatibility group (VCG) representative isolates by DNA fingerprinting analysis using primer ILOE. The isolate accession numbers or VCG codes and DNA fingerprint group (DFG) numbers are indicated at the top of the figure. The sizes in base pairs of the molecular weight marker are indicated to the left of the figure.

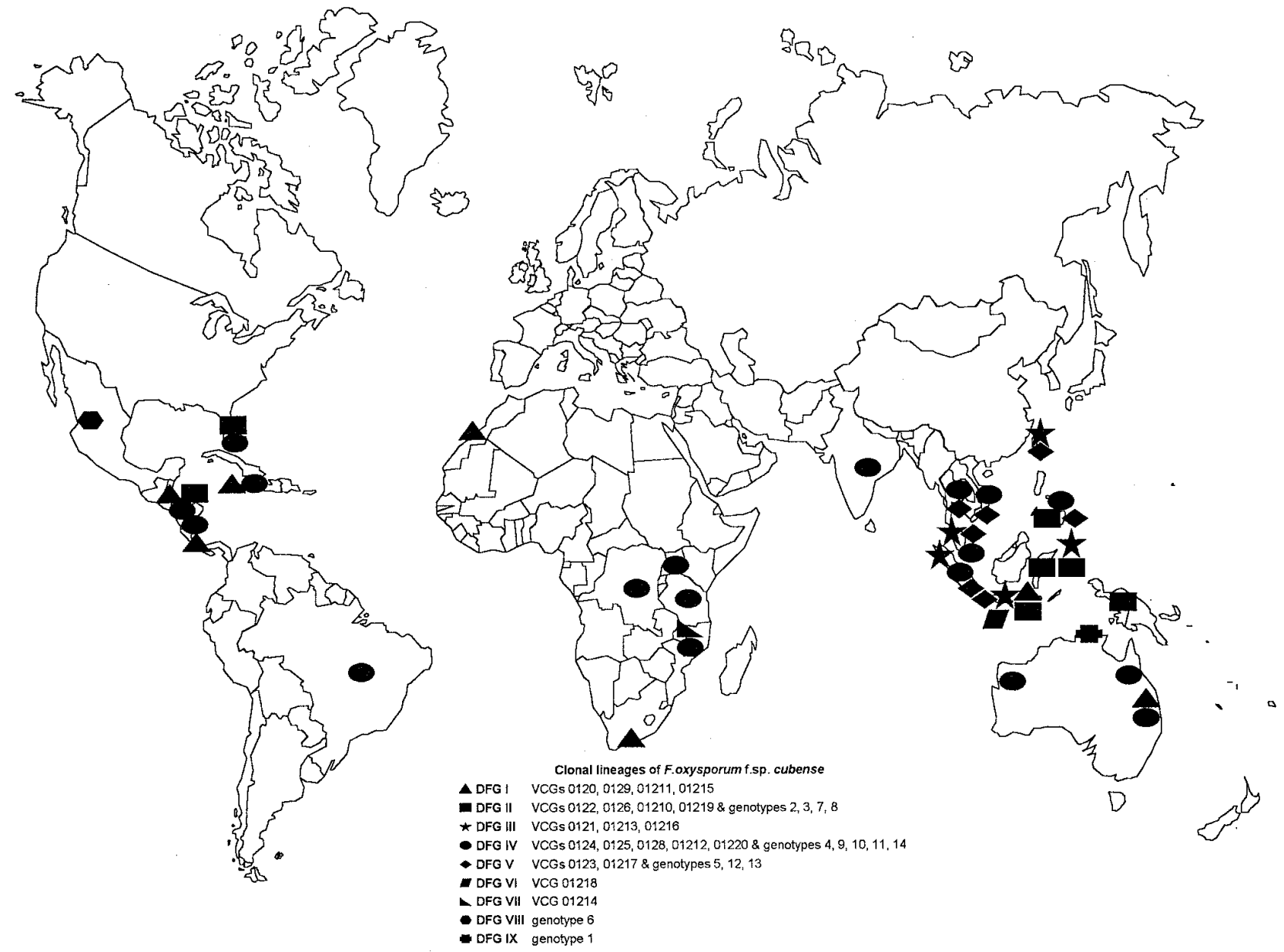

Fig. 4. Global distribution of DNA fingerprint group (DFG) lineages within Fusarium oxysporum f. sp. cubense. 
In this study, we have further differentiated the two major groups within $F$. oxysporum f. sp. cubense into nine clonal lineages based on analysis of more isolates (a total of 341) and more molecular markers (a total of 483) than previous studies. There was broad agreement between the lineages based on DNA fingerprinting analysis and those of Koenig et al. (10) based on RFLP analysis. Koenig et al. (10) described 10 clonal lineages within $F$. oxysporum f. sp. cubense based on RFLP analysis using anonymous singlecopy probes. Among 165 isolates, 72 haplotypes were identified, five of which accounted for nearly half the isolates examined. In this study, we have analyzed an additional five VCGs not studied by Koenig et al. (10) and 133 isolates not belonging to the currently defined VCGs. FOC I described by Koenig et al. (10) corresponded to DFG IV, except Koenig et al. (10) found that VCG 01212 was genetically distinct (FOC VIII) from VCGs 0124, 0125, and 0128 , but we found that VCG 01212 was $85 \%$ genetically similar to these VCGs. FOC II (VCGs 0120, 0126, 0129, and 01215) and FOC IX (VCG 01211) (10) corresponded to DFG I (VCGs 0120, 0129, 01211, and 01215) except for VCG 0126. We grouped VCG 0126 in DFG II along with VCGs 0122, 01210, and 01219, but Koenig et al. (10) classified VCGs 0122 and 01210 as distinct clades (FOC IV and VI, respectively) based on RFLP analysis. We found that VCGs 0121, 01213, and 01216 formed a distinct cluster (genetic similarity of $88 \%$ ); however, Koenig et al. (10) found that VCGs 0121 and 01213, together with three isolates from VCG 0120/01215, formed a clade (FOC III) of weak bootstrap support (53\%) that could not be confidently differentiated from several other VCGs. In agreement with Koenig et al. (10), we also found VCG 01214 (FOC V and DFG VII) to represent a genetically distinct population. Koenig et al. (10) found that the seven isolates they analyzed from VCG 0123 fell into either of two clades (FOC VII and FOC X), and the multilocus haplotype of these isolates appeared to represent a combination of alleles from the two main lineages, FOC I and II. In contrast, we analyzed 23 isolates belonging in VCG 0123 and found no genetic variation in the DNA fingerprints of these isolates. Isolates in VCG 0123 were found to be closely related to isolates in VCG 01217 , and several new genotypes including the isolates from the wild banana plants (M. acuminata subsp. malaccensis). Also in contrast to Koenig et al. (10), we did not find VCGs or genotypes common to more than one lineage.

Relationships between clonal lineages and races. The lineages within $F$. oxysporum f. sp. cubense corresponded with pathogenic race in that DFGs I to III contained all race 4 isolates, DFGs IV to VIII contained all race 1 and 2 isolates, and DFG IX was represented by race 3 isolates. A notable exception were the isolates in VCG 01220, which attacked Cavendish cultivar Williams in Carnarvon, Western Australia (18). Although these isolates were assigned to race 4 because they attacked Cavendish cultivars, they were more similar to race 1 and 2 isolates based on volatile, pectic enzyme and DNA fingerprinting analysis (18). The Cavendish plants were thought to be predisposed to infection by both waterlogging and drought stress. Race designation with this host-pathogen interaction is extremely difficult because of the inherent variability of the pathosystem as well as the influence of climate, edaphic conditions, and type of planting material used. For example, in subtropical regions such as Australia and South Africa, isolates in VCG 0120 are designated race 4, as they are capable of affecting Cavendish cultivars; however in tropical regions such as Costa Rica and Honduras, VCG 0120 isolates do not cause wilt in Cavendish cultivars and are, therefore, referred to as race 1. Consequently, isolates of the same genotype are classified as different races. In this study, isolates with virulence to Cavendish cultivars in the subtropics were restricted to DFG I, and isolates with virulence to Cavendish cultivars in the tropics to DFG III. The absence of Cavendish-virulent strains from the other lineages of $F$. oxysporum f. sp. cubense suggests that race 4 has not evolved from races 1 or 2 , as previously suggested (20). The genetic distance among the races suggests that races 1 and 2 have evolved together, whereas race 3 and race 4 are both of separate origin.

It is not known if the Australian isolates from Heliconia are a unique strain or the same strain that originated in Central America (32) that was perhaps introduced to Australia with infected planting material. No cultures of the original Central American strain were available for analysis, and more recent attempts to isolate $F$. oxysporum f. sp. cubense from Heliconia in tropical America have been unsuccessful. The original strains of race 3 were deliberately sought from Heliconia in Latin America as the possible origin of Fusarium wilt in banana ('Gros Michel'), after it was found that the bacterium that causes Moko disease of banana (Ralstonia solanacearum race 2) had originated in Heliconia (7). It would be interesting to reisolate $F$. oxysporum $\mathrm{f}$. sp. cubense from Heliconia in the tropical American jungle for comparison with the Australian isolates. Further examination of isolates from remnant plants of 'Gros Michel', 'Silk', and 'Bluggoe' in these jungle areas is also warranted.

Origin of $\boldsymbol{F}$. oxysporum $\mathbf{f}$. sp. cubense. There are two hypotheses for the origin of $F$. oxysporum f. sp. cubense. The first hypothesis proposed that the pathogen coevolved with banana in Asia and has been distributed to other countries in infected banana rhizomes and attached soil $(27,28,30)$. The second hypothesis is that the pathogen evolved independently from local populations of $F$. oxysporum in different countries to attack an introduced host plant (24). Our results indicate that while most lineages of $F$. oxysporum $\mathrm{f}$. sp. cubense have probably coevolved with banana in Asia, several lineages have probably arisen independently. The genetic isolation and limited geographic distribution of DFGs VI, VII, VIII, and IX indicate they have probably developed independently both within (DFG VI) and outside of (DFGs VII, VIII, and IX) the center of origin of the host.

If the coevolution hypothesis is correct, it is expected that there will be greatest diversity within populations of the pathogen at the center of origin of the host (31). In this study, DFGs II, IV, and V were the most divergent. Although DFG IV contained the most genotypes, the genotypes within this lineage were closely related, as evidenced by the similarity of their DNA fingerprint patterns and the presence of cross-compatible isolates among the VCGs. In contrast, there was greatest variability within DFGs II and V based on their DNA fingerprint patterns and also the absence of crosscompatible isolates among the different VCGs and genotypes within each of these lineages. Isolates in DFGs II and V were predominantly from Indonesia, Malaysia, and the Philippines (the exception was VCG 01210, which was unique to Florida). The fact that the most variable genotypes were of Asian origin supports the hypothesis of coevolution and subsequent distribution to other countries in infected banana rhizomes or attached soil (27). Further evidence that suggests $F$. oxysporum f. sp. cubense has coevolved with banana in Asia is the genetic similarity (95\%) between the Malaysian isolates (genotype 5) from the wilted wild banana plants (M. acuminata subsp. malaccensis) and isolates in DFG V. Besides these three affected plants of this species, no disease was observed (based on external symptoms) in the many other wild banana plants surveyed by some of the authors in the jungles of Indonesia, Malaysia, Thailand, and Viet Nam. This suggests either that the pathogen was not present or, more likely, that $F$. oxysporum $\mathrm{f}$. sp. cubense was present but was not capable of causing disease in the wild banana hosts. Gordon and Martyn (9) described F. oxysporum as nonpathogenic in native situations. If pathogenesis does develop in a native plant community, it is expected to be short-lived, because the fungus faces renewed competition from other microorganisms and the search for a new host is restricted spatially (9). More information on the interaction between $F$. oxysporum $\mathrm{f}$. sp. cubense and wild banana hosts would be obtained by analyzing more isolates from the wild pathosystem including isolates from the roots and rhizome of symptomless wild banana plants. The coevolution hypothesis has important implications in the selection 
of banana cultivars with resistance to Fusarium wilt, as resistant cultivars are most likely to be present in regions where there is greatest diversity within the host and the pathogen (31). It is apparent that further collection and genetic analysis of $F$. oxysporum f. sp. cubense strains from Asia, the center of origin and domestication of Musa, is necessary.

In summary, we have identified 33 different genotypes within $F$. oxysporum f. sp. cubense. Knowledge of the host range and geographic distribution of these genotypes may facilitate the selection of resistant cultivars for particular locations, depending on the endemic strains present. The known geographic distribution of these genotypes may also be useful in establishing quarantine zones to limit the spread, both nationally and internationally, of the more virulent strains of the pathogen.

\section{ACKNOWLEDGMENTS}

We thank the Australian Centre for International Agricultural Research, the Banana Industry Committee (New South Wales, Australia), the Banana Industry Protection Board (Queensland, Australia), the Cooperative Research Centre for Tropical Plant Pathology, the Horticultural Research and Development Corporation, the International Bank for Reconstruction and Development, the International Network for the Improvement of Banana and Plantain, and the Queensland Fruit and Vegetable Growers for funding this research. We thank A. Drenth and K. Goulter for helpful discussions and reviewing this manuscript.

\section{LITERATURE CITED}

1. Bentley, S., and Bassam, B. J. 1996. A robust DNA amplification fingerprinting system applied to analysis of genetic variation within Fusarium oxysporum f.sp. cubense. J. Phytopathol. 144:207-213.

2. Bentley, S., Pegg, K. G., and Dale, J. L. 1994. Optimization of RAPDPCR fingerprinting to analyse genetic variation within populations of Fusarium oxysporum f.sp. cubense. J. Phytopathol. 142:64-78.

3. Bentley, S., Pegg, K. G., and Dale, J. L. 1995. Genetic variation among a world-wide collection of isolates of Fusarium oxysporum f.sp. cubense analysed by RAPD-PCR fingerprinting. Mycol. Res. 99:1378-1384.

4. Boehm, E. W. A., Ploetz, R. C., and Kistler, H. C. 1994. Statistical analysis of electrophoretic karyotype variation among vegetative compatibility groups of Fusarium oxysporum f. sp. cubense. Mol. Plant-Microbe Interact. 7:196-207.

5. Brake, V. M., Pegg, K. G., Irwin, J. A. G., and Langdon, P. W. 1990. Vegetative compatibility groups within Australian populations of $\mathrm{Fu}$ sarium oxysporum f.sp. cubense, the cause of Fusarium wilt of bananas. Aust. J. Agric. Res. 41:863-870.

6. Brandes, E. W. 1919. Banana wilt. Phytopathology 9:339-390.

7. Buddenhagen, I. W. 1987. Disease susceptibility and genetics in relation to breeding of bananas and plantains. Pages 95-109 in: Banana and Plantain Breeding Strategies. G. J. Persley and E. A. De Langhe, eds. Australian Centre for International Agricultural Research (ACIAR) Proc. No. 21.

8. Caetano-Anollés, G., Bassam, B. J., and Gresshoff, P. M. 1991. DNA amplification fingerprinting using very short arbitrary oligonucleotide primers. Bio/Technology 9:553-557.

9. Gordon, T. R., and Martyn, R. D. 1997. The evolutionary biology of Fusarium oxysporum. Annu. Rev. Phytopathol. 35:111-128.

10. Koenig, R. L., Ploetz, R. C., and Kistler, H. C. 1997. Fusarium oxysporum f. sp. cubense consists of a small number of divergent and globally distributed clonal lineages. Phytopathology 87:915-923.

11. Miao, V. P. W. 1990. Using karyotype variability to investigate the origins and relatedness of isolates of Fusarium oxysporum f. sp. cubense. Pages 55-62 in: Fusarium Wilt of Banana. R. C. Ploetz, ed. The American Phytopathological Society, St. Paul, MN.

12. Moore, N. Y. 1994. Fusarium wilt of banana: Pathogen variability and host pathogen interaction. Ph.D. thesis. The University of Queensland, Brisbane, Australia.

13. Moore, N. Y., Hargreaves, P. A., Pegg, K. G., and Irwin, J. A. G. 1991. Characterisation of strains of Fusarium oxysporum f.sp. cubense by production of volatiles. Aust. J. Bot. 39:161-166.

14. Moore, N. Y., Pegg, K. G., Allen, R. A., and Irwin, J. A. G. 1993. Vegetative compatibility and distribution of Fusarium oxysporum f.sp. cubense in Australia. Aust. J. Exp. Agric. 33:797-802.

15. Moore, N. Y., Pegg, K. G., Langdon, P. W., Smith, M. K., and Whiley, A. W. 1993. Current research on Fusarium wilt of banana in Australia. Pages 270-284 in: Proc. Int. Symp. Recent Developments Banana Cultivation Tech. R. V. Valmayor, S. C. Hwang, R. C. Ploetz, S. W. Lee, and N. V. Roa, eds. International Network for the Improvement of Banana and Plantain/The Asia and Pacific Network (INIBAP/ASPNET), Los Banos, Laguna, Philippines.

16. O'Donnell, K., Kistler, H. C., Cigelnik, E., and Ploetz, R. C. 1998. Multiple evolutionary origins of the fungus causing Panama disease of banana: Concordant evidence from nuclear and mitochondrial gene genealogies. Proc. Natl. Acad. Sci. U.S.A. 95:2044-2049.

17. Pegg, K. G., Moore, N. Y., and Sorensen, S. 1993. Fusarium wilt in the Asian Pacific region. Pages 255-269 in: Proc. Int. Symp. Recent Developments Banana Cultivation Tech. R. V. Valmayor, S. C. Hwang, R. C. Ploetz, S. W. Lee, and N. V. Roa, eds. International Network for the Improvement of Banana and Plantain/The Asia and Pacific Network (INIBAP/ ASPNET), Los Banos, Laguna, Philippines.

18. Pegg, K. G., Shivas, R. G., Moore, N. Y., and Bentley, S. 1995. Characterisation of a unique population of Fusarium oxysporum f.sp. cubense causing Fusarium wilt in Cavendish bananas at Carnarvon, Western Australia. Aust. J. Agric. Res. 46:167-178.

19. Persley, G. J., and De Langhe, E. A. 1987. Summary of Discussions and Recommendations. Pages 9-17 in: Banana and Plantain Breeding Strategies. Australian Centre for International Agricultural Research (ACIAR) Proc. No. 21.

20. Ploetz, R. C. 1990. Population biology of Fusarium oxysporum f. sp. cubense. Pages 63-76 in: Fusarium Wilt of Banana. R. C. Ploetz, ed. The American Phytopathological Society, St. Paul, MN.

21. Ploetz, R. C. 1992. Fusarium wilt of banana (Panama disease). Pages 270-282 in: Diseases of Fruit Crops, Vol. III. J. Kumar, H. S. Chaube, U. S. Singh, and A. N. Mukhopadhyay, eds. Prentice Hall, Englewood Cliffs, NJ.

22. Ploetz, R. C., and Correll, J. C. 1988. Vegetative compatibility among races of Fusarium oxysporum f. sp. cubense. Plant Dis. 72:325-328.

23. Ploetz, R. C., Herbert, J., Sebasigari, K., Hernandez, J. H., Pegg, K. G., Ventura, J. A., and Mayato, L. S. 1990. Importance of Fusarium wilt in different banana-growing regions. Pages 9-26 in: Fusarium Wilt of Banana. R. C. Ploetz, ed. The American Phytopathological Society, St. Paul, MN.

24. Simmonds, N. W. 1966. Bananas. 2nd ed. Longmans, London.

25. Snyder, W. C., and Hansen, H. N. 1940. The species concept in Fusarium. Am. J. Bot. 27:64-67.

26. Stover, R. H. 1962. Studies on Fusarium wilt of banana. VIII. Differentiation of clones by cultural interactions and volatile substances. Can. J. Bot. 40:1467-1471.

27. Stover, R. H. 1962. Fusarial wilt (Panama disease) of bananas and other Musa species. Phytopathology Paper No. 4. Commonwealth Mycological Institute, Kew, Surrey, England.

28. Stover, R. H., and Buddenhagen, I. W. 1986. Banana breeding: Polyploidy, disease resistance and productivity. Fruits 41:175-191.

29. Stover, R. H., and Simmonds, N. W. 1987. Bananas. 3rd ed. Longmans, London.

30. Vakili, N. G. 1965. Fusarium wilt resistance in seedlings and mature plants of Musa species. Phytopathology 55:135-140.

31. Vavilov, N. I. 1922. The law of homologous series in variation. J. Genet. 12:47-89.

32. Waite, B. H. 1963. Wilt of Heliconia spp. caused by Fusarium oxysporum f.sp. cubense race 3. Trop. Agric. Trinidad 40:299-305.

33. Williams, J. G. K., Kubelik, A. R., Livak, K. J., Rafalski, J. A., and Tingey, S. V. 1990. DNA polymorphisms amplified by arbitrary primers are useful as genetic markers. Nucleic Acids Res. 18:6531-6535. 